\title{
Compton-like scattering of a scalar particle with $N$ photons and one graviton
}

\author{
Naser Ahmadiniaz ${ }^{\mathrm{a}, *}$, Filippo Maria Balli ${ }^{\mathrm{b}}$, Olindo Corradini ${ }^{\mathrm{b}, c, \mathrm{~d}, *}$, \\ José Manuel Dávila ${ }^{\mathrm{e}}$, Christian Schubert ${ }^{\mathrm{d}, \mathrm{f}}$ \\ ${ }^{a}$ Helmholtz-Zentrum Dresden-Rossendorf, Bautzner Landstraße 400, 01328 Dresden, Germany \\ b Dipartimento di Scienze Fisiche, Informatiche e Matematiche, Università degli Studi di Modena e Reggio Emilia, Via \\ Campi 213/A, I-41125 Modena, Italy \\ c INFN, Sezione di Bologna, Via Irnerio 46, I-40126 Bologna, Italy \\ d Max-Planck-Institut für Gravitationphysik, Albert-Einstein-Institut, Am Mühlenberg 1, 14476 Golm, Germany \\ e Facultad de Ciencias, Universidad Autónoma del Estado de México, Instituto Literario 100, C.P. 50000, Toluca, \\ Mexico \\ ${ }^{\mathrm{f}}$ Instituto de Física y Matemáticas, Universidad Michoacana de San Nicolás de Hidalgo, Edificio C-3, Apdo. Postal \\ 2-82, C.P. 58040, Morelia, Michoacán, Mexico
}

Received 29 October 2019; accepted 25 November 2019

Available online 27 November 2019

Editor: Stephan Stieberger

To the memory of Corneliu Sochichiu

\begin{abstract}
Tree-level scattering amplitudes for a scalar particle coupled to an arbitrary number $N$ of photons and a single graviton are computed. We employ the worldline formalism as the main tool to compute the irreducible part of the amplitude, where all the photons and the graviton are directly attached to the scalar line, then derive a "tree replacement" rule to construct the reducible parts of the amplitude which involve irreducible pure $N$-photon two-scalar amplitudes where one photon line emits the graviton. We test our construction by verifying the on-shell gauge and diffeomorphism Ward identities, at arbitrary $N$.

(c) 2019 Published by Elsevier B.V. This is an open access article under the CC BY license (http://creativecommons.org/licenses/by/4.0/). Funded by SCOAP ${ }^{3}$.

\footnotetext{
* Corresponding authors.

E-mail addresses: n.ahmadiniaz@hzdr.de (N. Ahmadiniaz), filippo.balli@unimore.it (F.M. Balli), olindo.corradini@unimore.it (O.Corradini),bcsdu@yahoo.com (J.M. Dávila), schubert@ifm.umich.mx (C. Schubert).
} 


\section{Contents}

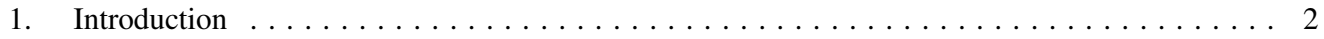

2. $\quad N$-photon scalar propagator from the worldline formalism $\ldots \ldots \ldots \ldots \ldots \ldots \ldots \ldots$

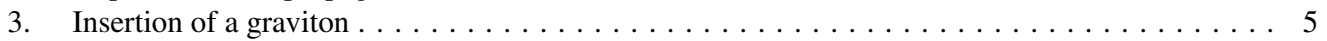

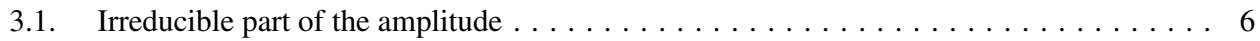

3.2. Reducible part of the amplitude . . . . . . . . . . . . . . . . 9

3.3. On-shell factorization property for the graviton photoproduction amplitude $\ldots \ldots \ldots \ldots 11$

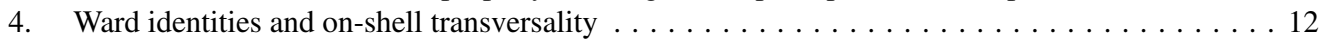

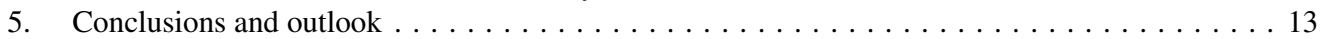

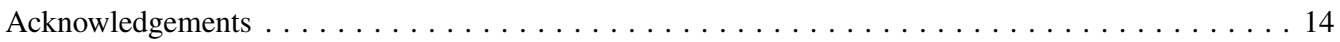

Appendix A. Two-photon one-graviton scalar propagator $\ldots \ldots \ldots \ldots \ldots \ldots \ldots \ldots \ldots \ldots$

Appendix B. Transversality of the amplitudes with one graviton and $N \leq 2$ photons $\ldots \ldots \ldots 16$

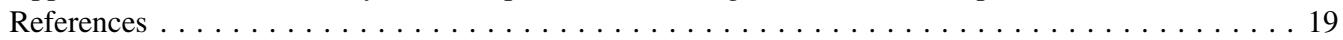

\section{Introduction}

The systematic computation of various classes of on-shell scattering amplitudes has become a very active field of research in the past few decades, and several very efficient methods have been put forward, which involve spinor helicity formalism, on-shell recursion relations, Ward identities, KLT relations, just to name a few-see [1] for a review. The main paradigm consists in avoiding the use of lagrangian field theories with all their plethoric structures and rely instead on more basic features (symmetries, kinematics,...) that allow to more efficiently compute the amplitudes. One feature which is common to most of these very effective methods-particularly those where the spinor helicity tricks are used as the main tool-is the masslessness of the propagating particles. As a complementary method in the present manuscript we consider a worldline approach, which instead ideally works with massive propagating particles.

Historically, the first pioneering work on the worldline approach to Quantum Field Theory is due to Feynman, who proposed a particle path integral representation for the dressed propagator of a scalar field coupled to electromagnetism [2]. However, this formulation was not taken seriously as an alternative to the standard Feynman diagram method for the actual computation of effective actions and scattering amplitudes until the early nineties, when Bern and Kosower [3, 4] derived novel rules for the construction of one-loop $N$-gluon amplitudes from first-quantized open string theory, and similar rules were shortly later derived from the closed string for one-loop $N$-graviton amplitudes [5].

For the gluonic case, these rules were then rederived from point particle path integrals by Strassler [6], which established this "worldline formalism" as a serious alternative to Feynman diagrams, and triggered a host of generalizations to other types of amplitudes and effective actions - see Ref. [7] for an earlier account on the development of the method. So far, the majority of developments and applications of the worldline approach have been at the loop level: multiloop calculations [8,9], worldline methods with strong external fields [10-13], the worldline formalism in curved spacetime [14], one-loop quantum gravity [15,16] and photon-graviton mixing in an electromagnetic field [17], the worldline Monte-Carlo approach to the Casimir effect [18], higher-spin field theory [19,20], and applications to QFT on manifolds with boundary [21,22], 
noncommutative QFT's [23] and form-factor decompositions of off-shell gluon amplitudes [24, 25] and many more.

On other hand, the worldline approach to dressed propagators and to the associated scattering amplitudes is a much less developed subject of research, though the Bern-Kosower rules for a scalar particle line coupled to electromagnetism in vacuum were found soon after their one-loop counterparts [26,27]. However, more recently, master formulas for a scalar particle in a constant background field were derived [28], and the coupling to non-abelian fields in vacuum was also studied [29]. Generalizations to propagators of fields with spin are even more rare. The straightforward procedure would be to consider locally supersymmetric spinning particle models on the worldline [31] — recently also Einstein gravity was studied through the BRST quantization of an $\mathcal{N}=4$ spinning particle model [32]. However, there are technical difficulties to be overcome in the path integral quantization of such models on the open line, since the gravitino present in the locally supersymmetric model cannot be completely gauged away, and the coherent state boundary conditions for the fermionic coordinates, responsible for providing the spinorial degrees of freedom to the particle, do not appear to be very convenient. A suitable alternative approach is to employ the 'Symb' map developed in [30,33] which reproduces the spin-factor potential in terms of fermionic coordinates with antiperiodic boundary conditions, and the resulting particle models are now globally supersymmetric. This approach allowed to compute some previously neglected one-particle reducible contributions to the fermion propagator in a constant field [34]. Moreover, a derivation of a master formula for the tree level $N$-photon fermion propagator is under completion [35].

In the present manuscript we instead take a path towards the derivation of tree level amplitudes with gravitons, using as a main tool the worldline approach in curved space. In fact, at the level of one photon-i.e. for the gravitational photoproduction process - the amplitude displays a very interesting factorization property [36-41], which is briefly reviewed below in a dedicated subsection. However, this nice factorization property appears not to work beyond the $N=1$ case since there are too few conservation laws—-see [36,37] for a detailed discussion. Here, we consider a scalar particle line perturbatively coupled to electromagnetism and to gravity, and provide a master formula which involves the inclusion of $N$ photons and one graviton into the scalar line, i.e. we add a graviton to Daikouji et al.'s formula [26] which was also rederived in [27]. The inclusion even of a single graviton is by no means trivial for various reasons. Firstly, it boils down to the application of the worldline formalism in curved space which, although being well understood by now, is certainly trickier than its flat space counterpart. In fact, the coupling to gravity, in the perturbative approach requires the use of regularization schemes and careful treatment of the non-trivial path integral measure [42]. Moreover, the graviton can either couple directly to the scalar line, but it may also be emitted from a photon line, since gravity couples to the photon stress tensor. This second contribution involves diagrams that are one-particle reducible in the photon lines, and akin to what occurs in the presence of a non-abelian gauge field where, say, a gluon emitted from the scalar line can split in two or three gluons. Similar issues were indeed already discussed, for instance in the worldgraph approach to Yang-Mills amplitudes [43] and in worldline calculations [44].

A particularly elegant feature of the original Bern-Kosower and Bern-Dunbar-Shimada rules for gluon and graviton amplitudes is that they provide a simple rule for constructing the reducible contributions from the irreducible ones at the integrand level, instead of the usual "sewing trees onto loops" procedure. Here, we provide a similar novel replacement rule, which allows us to obtain the reducible part of the amplitudes with the graviton in terms of the scalar lines with only photons attached, thus in terms of amplitudes for which a convenient generating master formula 
exists. For this purpose, it will be essential that in the worldline approach to scattering amplitudes there is a priori no need to impose on-shell conditions on the external lines.

In the following we first rederive the $N$-photon scalar propagator and the associated master formula, since it is one of our main tools. Then we consider the insertion of a graviton and single out the irreducible part of the amplitudes - by using a helpful parametrization of the graviton polarization, and the reducible part through the aforementioned replacement rule. This allows us to give a compact formula for the full tree level amplitude with $N$ photons, one graviton and two scalars. We thus test our master formula by checking the on-shell transversality on the photon lines and graviton lines. In the graviton case, this requires a conspiration between the reducible and irreducible contributions that becomes rather transparent in our approach. Some computational details, concerning amplitudes with $N \leq 2$, are relegated to the appendix.

\section{2. $N$-photon scalar propagator from the worldline formalism}

The photon-dressed propagator in scalar QED can be efficiently obtained using the line path integral of a scalar particle in the presence of an external electromagnetic field,

$$
\left\langle\phi\left(x^{\prime}\right) \bar{\phi}(x)\right\rangle_{A}=\int_{0}^{\infty} d T e^{-m^{2} T} \int_{x(0)=x}^{x(T)=x^{\prime}} D x e^{-\int_{0}^{T} d \tau\left(\frac{1}{4} \dot{x}^{2}+i e \dot{x} \cdot A(x)\right)} .
$$

The $N$-photon scalar propagator, i.e. the scalar propagator with the insertion of $N$ photons can be obtained with the straightforward recipe that we briefly review here. Firstly, write the external field as a sum of $N$ photons

$$
A_{\mu}(x(\tau))=\sum_{l=1}^{N} \varepsilon_{l, \mu} e^{i k_{l} \cdot x},
$$

then extract from (1) the multi-linear part, in the various polarizations $\varepsilon_{l}$, and Fourier transform in the two external scalar lines. This leads to

$$
\begin{aligned}
D^{(N)}\left(p, p^{\prime} ; \varepsilon_{1}, k_{1}, \ldots, \varepsilon_{N}, k_{N}\right) & =(-i e)^{N} \int_{0}^{\infty} d T e^{-m^{2} T} \int d^{4} x \int d^{4} x^{\prime} e^{i\left(p \cdot x+p^{\prime} \cdot x^{\prime}\right)} \\
& \times \int_{x(0)=x}^{x(T)=x^{\prime}} D x e^{-\int_{0}^{T} d \tau \frac{1}{4} \dot{x}^{2}} \prod_{l=1}^{N} \int_{0}^{T} d \tau_{l} \varepsilon_{l} \cdot \dot{x}\left(\tau_{l}\right) e^{i k_{l} \cdot x\left(\tau_{l}\right)} .
\end{aligned}
$$

It is thus convenient to split the particle path in terms of a background $\bar{x}^{\mu}(\tau)=x^{\mu}+\left(x^{\prime \mu}-x^{\mu}\right) \frac{\tau}{T}$ and fluctuations $q^{\mu}(\tau)$ with vanishing boundary conditions. One thus gets

$$
\begin{aligned}
& D^{(N)}\left(p, p^{\prime} ; \varepsilon_{1}, k_{1}, \ldots, \varepsilon_{N}, k_{N}\right) \\
& =(-i e)^{N} \int_{0}^{\infty} d T e^{-m^{2} T} \int d^{4} x \int d^{4} x^{\prime} e^{i\left(p \cdot x+p^{\prime} \cdot x^{\prime}\right)-\frac{1}{4 T}\left(x-x^{\prime}\right)^{2}} \\
& \quad \times\left. e^{\sum_{l}\left(i k_{l} \cdot x+\frac{\varepsilon_{l}}{T} \cdot\left(x^{\prime}-x\right)\right)} \int_{q(0)=0}^{q(T)=0} D q e^{-\int_{0}^{T} d \tau \frac{1}{4} \dot{q}^{2}} \prod_{l=1}^{N} \int_{0}^{T} d \tau_{l} e^{i k_{l} \cdot\left(\left(x^{\prime}-x\right) \frac{\tau_{l}}{T}+q\left(\tau_{l}\right)\right)+\varepsilon_{l} \cdot \dot{q}\left(\tau_{l}\right)}\right|_{\mathrm{m} .1 .},
\end{aligned}
$$


where 'm.l.' indicates that we are only meant to pick out the multilinear part in all the polarizations. The latter path integral thus provides the correlation function of the product of $N$ photon vertex operators

$$
V_{A}[\varepsilon, k]=\left.e^{i k \cdot x+\frac{\varepsilon}{T} \cdot\left(x^{\prime}-x\right)} \int_{0}^{T} d \tau e^{i k \cdot\left(\left(x-x^{\prime}\right) \frac{\tau}{T}+q(\tau)\right)+\varepsilon \cdot \dot{q}(\tau)}\right|_{\operatorname{lin}},
$$

with respect to the Gaussian measure $\int D q e^{-\frac{1}{4} \int \dot{q}^{2}}$, which has normalization $\frac{1}{(4 \pi T)^{D / 2}}$ and yields the Green's functions

$$
\begin{aligned}
& \left\langle q^{\alpha}(\tau) q^{\alpha^{\prime}}\left(\tau^{\prime}\right)\right\rangle=-2 \delta^{\alpha \alpha^{\prime}} \Delta\left(\tau, \tau^{\prime}\right), \\
& \Delta\left(\tau, \tau^{\prime}\right)=\frac{\tau \tau^{\prime}}{T}+\frac{1}{2}\left|\tau-\tau^{\prime}\right|-\frac{1}{2}\left(\tau+\tau^{\prime}\right) .
\end{aligned}
$$

Thus, after some straightforward algebra one finds the Bern-Kosower-like master formula originally obtained by Daikouji et al. [26] and later in the worldline formalism in [27], i.e.

$$
\begin{aligned}
& \widetilde{D}^{(N)}\left(p, p^{\prime} ; \varepsilon_{1}, k_{1}, \ldots, \varepsilon_{N}, k_{N}\right) \\
& =(-i e)^{N} \int_{0}^{\infty} d T e^{-T\left(m^{2}+p^{\prime 2}\right)} \prod_{l=1}^{N} \int_{0}^{T} d \tau_{l} \exp \left\{\left(p^{\prime}-p\right) \cdot \sum_{l=1}^{N}\left(-k_{l} \tau_{l}+i \varepsilon_{l}\right)\right. \\
& \left.\quad+\sum_{l, l^{\prime}=1}^{N}\left(k_{l} \cdot k_{l^{\prime}} \Delta_{l-l^{\prime}}-2 i \varepsilon_{l} \cdot k_{l^{\prime}} \dot{\Delta}_{l-l^{\prime}}+\varepsilon_{l} \cdot \varepsilon_{l^{\prime}} \ddot{\Delta}_{l-l^{\prime}}\right)\right\}\left.\right|_{\text {m.l. }},
\end{aligned}
$$

where

$$
\Delta_{l-l^{\prime}}:=\frac{1}{2}\left|\tau_{l}-\tau_{l^{\prime}}\right|
$$

is the translation-invariant part of (7). Above we have also stripped off the overall momentumconservation delta function. The Feynman amplitude for the tree-level scattering of two scalars and $N$ photons can thus be obtained from (8) by truncating the external scalar lines, i.e. multiplying by $\left(p^{2}+m^{2}\right)\left(p^{\prime 2}+m^{2}\right)$,

$$
\mathcal{D}^{(N)}\left(p, p^{\prime} ; \varepsilon_{1}, k_{1}, \ldots, \varepsilon_{N}, k_{N}\right)=\left(p^{2}+m^{2}\right)\left(p^{\prime 2}+m^{2}\right) \widetilde{D}^{(N)}\left(p, p^{\prime} ; \varepsilon_{1}, k_{1}, \ldots, \varepsilon_{N}, k_{N}\right) .
$$

Note that, as already mentioned in the Introduction, this expression holds off the mass-shell of the external particles. However, going on-shell leads to transversality in all the photon lines, upon the replacement $\varepsilon_{l}\left(k_{l}\right) \rightarrow k_{l}$, as will be reviewed below.

\section{Insertion of a graviton}

The computation of scattering amplitudes of the scalar particle with photons and gravitons, can be performed by considering the worldline representation in curved space [45]. For the propagator of a scalar particle minimally coupled to gravity, we have 


$$
\begin{aligned}
\left\langle\phi\left(x^{\prime}\right) \bar{\phi}(x)\right\rangle_{A, g} & =\int_{0}^{\infty} d T e^{-m^{2} T} \int_{x(0)=x}^{x(T)=x^{\prime}} D x D a D b D c \\
& \times e^{-\int_{0}^{T} d \tau\left(\frac{1}{4} g_{\mu \nu}(x)\left(\dot{x}^{\mu} \dot{x}^{\nu}+a^{\mu} a^{\nu}+b^{\mu} c^{\nu}\right)+i e \dot{x} \cdot A(x)\right)},
\end{aligned}
$$

where the fields $a^{\mu}$, and $b^{\mu}$ and $c^{\mu}$ are commuting, respectively anti-commuting, auxiliary fields (Lee-Yang ghosts) which were found to suitably represent the Einstein-invariant path integral measure, and have vanishing boundary conditions. By expanding the metric about the flat background

$$
g_{\mu \nu}(x)=\delta_{\mu \nu}+\kappa \epsilon_{\mu \nu} e^{i k_{0} \cdot x},
$$

and using the same split described above for the particle paths-we can read off the graviton vertex operator

$$
\begin{aligned}
V_{g}\left[\epsilon, k_{0}\right]= & e^{i k_{0} \cdot x+\frac{1}{T^{2}}\left(x^{\prime}-x\right) \cdot \epsilon \cdot\left(x^{\prime}-x\right)} \\
& \times\left.\int_{0}^{T} d \tau e^{i k_{0} \cdot\left(\left(x^{\prime}-x\right) \frac{\tau}{T}+q\right)+\epsilon \mu \nu\left(\frac{2}{T}\left(x^{\prime}-x\right)^{\mu} \dot{q}^{\nu}+\dot{q}^{\mu} \dot{q}^{\nu}+a^{\mu} a^{\nu}+b^{\mu} c^{\nu}\right)}\right|_{\operatorname{lin}},
\end{aligned}
$$

along with auxiliary fields propagators

$$
\begin{aligned}
& \left\langle a^{\mu}(\tau) a^{v}\left(\tau^{\prime}\right)\right\rangle=2 \delta^{\mu v} \delta\left(\tau, \tau^{\prime}\right), \\
& \left\langle b^{\mu}(\tau) c^{v}\left(\tau^{\prime}\right)\right\rangle=-4 \delta^{\mu v} \delta\left(\tau, \tau^{\prime}\right) .
\end{aligned}
$$

Hence, the irreducible part of the tree-level scalar propagator with the insertion of $N$ photons and one graviton reads

$$
\begin{aligned}
& D^{(N, 1)}\left(p, p^{\prime} ; \varepsilon_{1}, k_{1}, \ldots, \varepsilon_{N}, k_{N} ; \epsilon, k_{0}\right)=(-i e)^{N}\left(-\frac{\kappa}{4}\right) \int_{0}^{\infty} d T e^{-m^{2} T} \\
& \times \int d^{4} x \int d^{4} x^{\prime} e^{i\left(p \cdot x+p^{\prime} \cdot x^{\prime}\right)-\frac{1}{4 T}\left(x-x^{\prime}\right)^{2}} \frac{1}{(4 \pi T)^{\frac{D}{2}}}\left\langle\prod_{l=1}^{N} V_{A}\left[\varepsilon_{l}, k_{l}\right] V_{g}\left[\epsilon, k_{0}\right]\right\rangle,
\end{aligned}
$$

where only the part linear in all the polarizations ( $\varepsilon$ 's and $\epsilon$ ) has to be retained. In the next sections we provide a specific recipe to handle this task and obtain a useful master formula for the full Feynman amplitude.

\subsection{Irreducible part of the amplitude}

In order to explicitly compute the irreducible part of the $N$-photon one-graviton amplitude (see Fig. 1) we find it convenient to parametrize the graviton polarization as

$$
\begin{aligned}
& \epsilon_{\mu \nu}:=\lambda_{\mu} \rho_{\nu}, \\
& \varepsilon_{0 \mu}:=\lambda_{\mu}+\rho_{\mu},
\end{aligned}
$$

where, in Eq. (17), symmetrization between indices is implied. Such parametrization has to be understood as a simple book-keeping device to combine photon and graviton insertions together; 


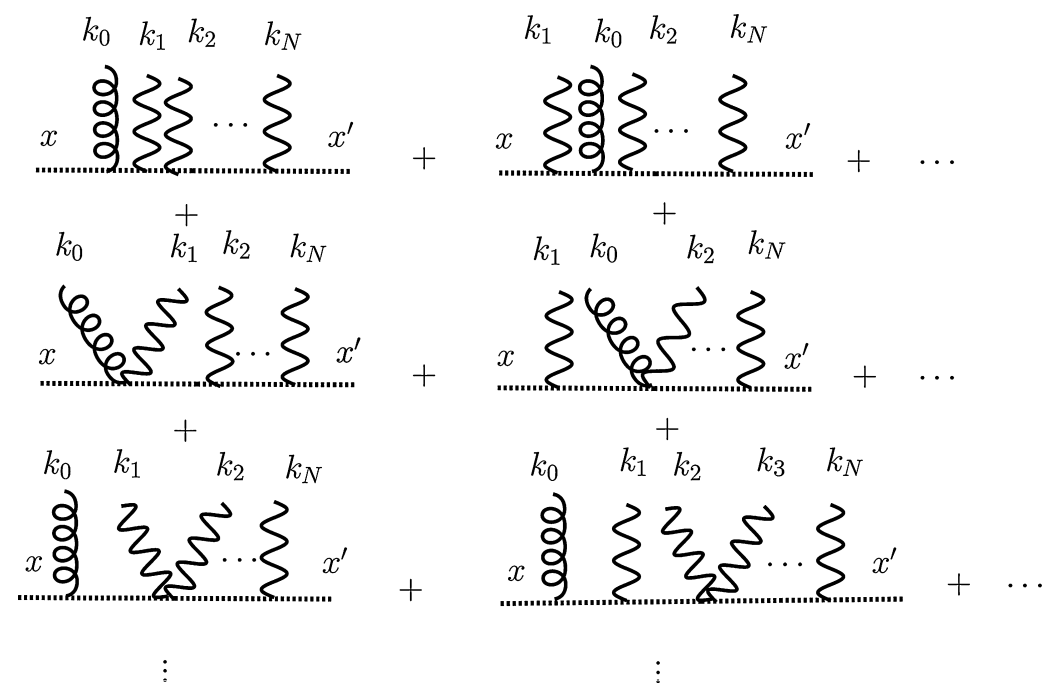

Fig. 1. The Feynman diagram representation (in configuration space) for irreducible contributions to $N$-photon onegraviton amplitude. The diagrams in the second and third lines involve quartic vertices that in the worldline approach come from delta functions, e.g. the first one is given by $\delta\left(\tau_{0}-\tau_{1}\right)$ etc.

at the end the graviton polarization is reconstructed from the term simultaneously linear in $\lambda$ and $\rho$. In fact, with a single graviton insertion, the ghost contribution cancels against the singular part of the $\left\langle\dot{q}^{\mu}\left(\tau_{0}\right) \dot{q}^{\nu}\left(\tau_{0}\right)\right\rangle$ propagator that appears in the graviton vertex operator. We can thus neglect the ghost contributions, provided we take $\left\langle\dot{q}^{\mu}\left(\tau_{0}\right) \dot{q}^{\nu}\left(\tau_{0}\right)\right\rangle \cong-\frac{2}{T} \delta^{\mu \nu}$ in the graviton sector. The graviton vertex operator can thus be written as

$$
V_{g}\left[\epsilon, k_{0}\right]=\left.e^{i k_{0} \cdot x+\frac{\varepsilon_{0}}{T} \cdot\left(x^{\prime}-x\right)} \int_{0}^{T} d \tau_{0} e^{i k_{0} \cdot\left(\left(x^{\prime}-x\right) \frac{\tau_{0}}{T}+q\left(\tau_{0}\right)\right)+\varepsilon_{0} \cdot \dot{q}\left(\tau_{0}\right)}\right|_{\text {lin. } \lambda, \rho},
$$

which has the same form as the photon counterpart, with the only subtlety that the linear part in $\lambda$ and $\rho$ comes from the quadratic part in $\varepsilon_{0}$. We thus get the " $N$-photon one-graviton scalar propagator"

$$
\begin{aligned}
& \widetilde{D}^{(N, 1)}\left(p, p^{\prime} ; \varepsilon_{1}, k_{1}, \ldots, \varepsilon_{N}, k_{N} ; \epsilon, k_{0}\right) \\
& =(-i e)^{N}\left(-\frac{\kappa}{4}\right) \int_{0}^{\infty} d T e^{-T\left(m^{2}+p^{\prime 2}\right)} \prod_{l=0}^{N} \int_{0}^{T} d \tau_{l} \exp \left\{\left(p^{\prime}-p\right) \cdot \sum_{l=0}^{N}\left(-k_{l} \tau_{l}+i \varepsilon_{l}\right)\right. \\
& \left.\quad+\sum_{l<l^{\prime}=0}^{N}\left(k_{l} \cdot k_{l^{\prime}}\left|\tau_{l}-\tau_{l^{\prime}}\right|+i\left(\varepsilon_{l^{\prime}} \cdot k_{l}-\varepsilon_{l} \cdot k_{l^{\prime}}\right) \operatorname{sgn}\left(\tau_{l}-\tau_{l^{\prime}}\right)+2 \varepsilon_{l} \cdot \varepsilon_{l^{\prime}} \delta\left(\tau_{l}-\tau_{l^{\prime}}\right)\right)\right\}\left.\right|_{\mathrm{m} .1 .},
\end{aligned}
$$

where 'm.l.' stands for 'multilinear' i.e. linear in all $\varepsilon_{l}, l=1, \ldots, N$ and linear in $\lambda$ and $\rho$, and with $\ddot{\Delta}_{0-0^{\prime}}=0$. On the mass shell of the scalar particle, upon truncation of the external scalar lines, the latter provides a contribution to the tree-level amplitude with $N$ photons, one graviton and two scalars that we will refer to as 'irreducible' 


$$
\begin{aligned}
& \mathcal{D}_{\text {irred }}^{(N, 1)}\left(p, p^{\prime} ; \varepsilon_{1}, k_{1}, \ldots, \varepsilon_{N}, k_{N} ; \epsilon, k_{0}\right) \\
& =\left(p^{2}+m^{2}\right)\left(p^{\prime 2}+m^{2}\right) \widetilde{D}^{(N, 1)}\left(p, p^{\prime} ; \varepsilon_{1}, k_{1}, \ldots, \varepsilon_{N}, k_{N} ; \epsilon, k_{0}\right),
\end{aligned}
$$

meaning that it cannot be divided into two subdiagrams by cutting a photon line or the graviton line.

Let us single out some special cases of the previous formula which will be helpful later. Let us begin considering the case $N=0$, i.e. the 'graviton-scalar' vertex,

$$
\widetilde{D}^{(0,1)}\left(p, p^{\prime} ; \epsilon, k_{0}\right)=\left.\left(-\frac{\kappa}{4}\right) \int_{0}^{\infty} d T e^{-T\left(m^{2}+p^{\prime 2}\right)} \int_{0}^{T} d \tau_{0} e^{\left(p^{\prime}-p\right) \cdot\left(-k_{0} \tau_{0}+i \varepsilon_{0}\right)}\right|_{\text {m.l. }},
$$

which, using momentum conservation, can be reduced to

$$
\widetilde{D}^{(0,1)}\left(p, p^{\prime} ; \epsilon, k_{0}\right)=\frac{\kappa}{4}\left(p^{\prime}-p\right)^{\mu} \epsilon_{\mu \nu}\left(p^{\prime}-p\right)^{v} \frac{1}{\left(p^{\prime 2}+m^{2}\right)\left(p^{2}+m^{2}\right)},
$$

and, upon truncation, leads to the amplitude (vertex)

$$
\mathcal{D}^{(0,1)}\left(p, p^{\prime} ; \epsilon, k_{0}\right)=\frac{\kappa}{4}\left(p^{\prime}-p\right)^{\mu} \epsilon_{\mu \nu}\left(p^{\prime}-p\right)^{\nu} .
$$

For $N=1$, the irreducible part of the gravitational photoproduction amplitude can be easily obtained from

$$
\begin{aligned}
& \widetilde{D}^{(1,1)}\left(p, p^{\prime} ; \varepsilon_{1}, k_{1} ; \epsilon, k_{0}\right)=(-i e)\left(-\frac{\kappa}{4}\right) \int_{0}^{\infty} d T e^{-T\left(m^{2}+p^{\prime 2}\right)} \int_{0}^{T} d \tau_{0} \int_{0}^{T} d \tau_{1} \\
& \times\left. e^{\left(p^{\prime}-p\right) \cdot\left(-k_{0} \tau_{0}-k_{1} \tau_{1}+i \varepsilon_{0}+i \varepsilon_{1}\right)} e^{k_{0} \cdot k_{1}\left|\tau_{0}-\tau_{1}\right|+i\left(\varepsilon_{1} \cdot k_{0}-\varepsilon_{0} \cdot k_{1}\right) \operatorname{sgn}\left(\tau_{0}-\tau_{1}\right)+2 \varepsilon_{0} \cdot \varepsilon_{1} \delta\left(\tau_{0}-\tau_{1}\right)}\right|_{\text {m.l. }},
\end{aligned}
$$

where the $\delta\left(\tau_{0}-\tau_{1}\right)$ part yields the seagull diagram, whereas the time ordered parts $\left(\tau_{0}>\tau_{1}\right.$ and $\tau_{0}<\tau_{1}$ ) yield the diagrams where photon and graviton are singly emitted by the scalar line. We thus get the following irreducible contribution to the Feynman amplitude

$$
\begin{aligned}
& \mathcal{D}_{\text {irred }}^{(1,1)}\left(p, p^{\prime} ; \varepsilon_{1}, k_{1} ; \epsilon, k_{0}\right)=\left(p^{\prime 2}+m^{2}\right)\left(p^{2}+m^{2}\right) \widetilde{D}^{(1,1)}\left(p, p^{\prime} ; \varepsilon_{1}, k_{1} ; \epsilon, k_{0}\right) \\
& =e \kappa\left[\left(p-p^{\prime}\right) \cdot \epsilon \cdot \varepsilon_{1}+\frac{\varepsilon_{1} \cdot p^{\prime} p \cdot \epsilon \cdot p}{p \cdot k_{0}}-\frac{\varepsilon_{1} \cdot p p^{\prime} \cdot \epsilon \cdot p^{\prime}}{p \cdot k_{1}}\right] .
\end{aligned}
$$

Finally, let us consider the irreducible contribution to the two-photon one-graviton amplitude, which is obviously trickier than the previous ones, though the worldline approach allows to obtain a quite compact representation. We report here the final result (the interested reader will find details of the computation to the Appendix A) which reads

$$
\begin{aligned}
\mathcal{D}_{\text {irred }}^{(2,1)}\left(p, p^{\prime} ; \varepsilon_{1}, k_{1}, \varepsilon_{2}, k_{2} ; \epsilon, k_{0}\right) \\
=\kappa e^{2}\left\{2\left(\varepsilon_{1} \epsilon \varepsilon_{2}\right)-2 \frac{\varepsilon_{1} \cdot \varepsilon_{2}\left(p^{\prime} \epsilon p^{\prime}\right)}{m^{2}+\left(p^{\prime}+k_{0}\right)^{2}}-2 \frac{\varepsilon_{1} \cdot \varepsilon_{2}(p \epsilon p)}{m^{2}+\left(p+k_{0}\right)^{2}}\right. \\
\quad+2 \frac{\varepsilon_{1} \cdot p\left(\varepsilon_{2} \epsilon\left(p^{\prime}-p-k_{1}\right)\right)}{m^{2}+\left(p+k_{1}\right)^{2}}+2 \frac{\varepsilon_{1} \cdot p^{\prime}\left(\varepsilon_{2} \epsilon\left(p-p^{\prime}-k_{1}\right)\right)}{m^{2}+\left(p^{\prime}+k_{1}\right)^{2}} \\
\quad+2 \frac{\varepsilon_{2} \cdot p\left(\varepsilon_{1} \epsilon\left(p^{\prime}-p-k_{2}\right)\right)}{m^{2}+\left(p+k_{2}\right)^{2}}+2 \frac{\varepsilon_{2} \cdot p^{\prime}\left(\varepsilon_{1} \epsilon\left(p-p^{\prime}-k_{2}\right)\right)}{m^{2}+\left(p^{\prime}+k_{2}\right)^{2}}
\end{aligned}
$$




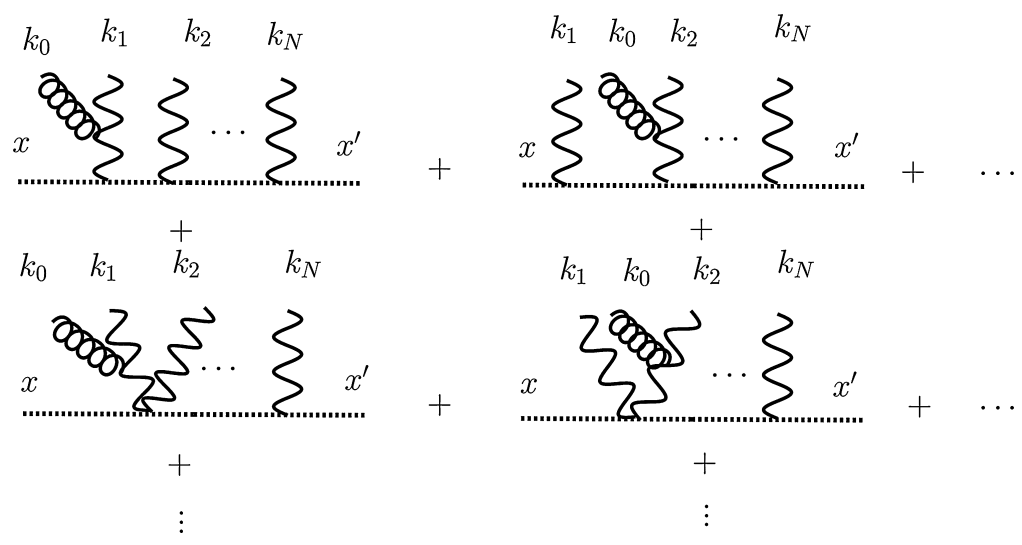

Fig. 2. Feynman diagram representation of the reducible contribution to $N$-photon one-graviton amplitude.

$$
\begin{aligned}
& +4 \frac{\left(p^{\prime} \epsilon p^{\prime}\right) \varepsilon_{1} \cdot\left(p+k_{2}\right) \varepsilon_{2} \cdot p}{\left(\left(p+k_{2}\right)^{2}+m^{2}\right)\left(\left(p^{\prime}+k_{0}\right)^{2}+m^{2}\right)}+4 \frac{\left(p^{\prime} \epsilon p^{\prime}\right) \varepsilon_{2} \cdot\left(p+k_{1}\right) \varepsilon_{1} \cdot p}{\left(\left(p+k_{1}\right)^{2}+m^{2}\right)\left(\left(p^{\prime}+k_{0}\right)^{2}+m^{2}\right)} \\
& +4 \frac{(p \epsilon p) \varepsilon_{1} \cdot\left(p^{\prime}+k_{2}\right) \varepsilon_{2} \cdot p^{\prime}}{\left(\left(p+k_{0}\right)^{2}+m^{2}\right)\left(\left(p^{\prime}+k_{2}\right)^{2}+m^{2}\right)}+4 \frac{(p \epsilon p) \varepsilon_{2} \cdot\left(p^{\prime}+k_{1}\right) \varepsilon_{1} \cdot p^{\prime}}{\left(\left(p+k_{0}\right)^{2}+m^{2}\right)\left(\left(p^{\prime}+k_{1}\right)^{2}+m^{2}\right)} \\
& \left.+4 \frac{\left(\left(p+k_{1}\right) \epsilon\left(p^{\prime}+k_{2}\right)\right) \varepsilon_{1} \cdot p \varepsilon_{2} \cdot p^{\prime}}{\left(\left(p+k_{1}\right)^{2}+m^{2}\right)\left(\left(p^{\prime}+k_{2}\right)^{2}+m^{2}\right)}+4 \frac{\left(\left(p+k_{2}\right) \epsilon\left(p^{\prime}+k_{1}\right)\right) \varepsilon_{2} \cdot p \varepsilon_{1} \cdot p^{\prime}}{\left(\left(p+k_{2}\right)^{2}+m^{2}\right)\left(\left(p^{\prime}+k_{1}\right)^{2}+m^{2}\right)}\right\} .
\end{aligned}
$$

In the next section we tackle the reducible part of the amplitude.

\subsection{Reducible part of the amplitude}

The external graviton can couple directly to the scalar line, as reproduced by the formulas described in the previous section, but it can also couple to the photon lines-see Fig. 2 for the diagrammatic representation of these contributions. From a field theory view point this is encoded in the vertex

$$
\mathcal{V}[A, h]=\frac{\kappa}{2} \int d^{4} x h_{\mu \nu} T^{\mu \nu}=\frac{\kappa}{2} \int d^{4} x h_{\mu \nu}\left(F^{\mu \alpha} F_{\alpha}^{\nu}-\frac{1}{4} \delta^{\mu \nu} F^{\alpha \beta} F_{\alpha \beta}\right),
$$

which, using the tracelessness of the on-shell graviton, leads to the following tree-level amplitude between two photons and one graviton

$$
\Gamma_{g \gamma \gamma}\left[\varepsilon, k, \varepsilon^{\prime}, k^{\prime} ; \epsilon, k_{0}\right]=\kappa\left[(k \epsilon k) \varepsilon \cdot \varepsilon^{\prime}+\left(\varepsilon \epsilon \varepsilon^{\prime}\right) k \cdot k_{0}-(\varepsilon \epsilon k) k \cdot \varepsilon^{\prime}-\left(k \epsilon \varepsilon^{\prime}\right) \varepsilon \cdot k_{0}\right],
$$

where $(a \epsilon b):=a_{\mu} \epsilon^{\mu \nu} b_{\nu}$, and we have used the transversality conditions $k_{0 \mu} \epsilon^{\mu \nu}=k_{\mu} \varepsilon^{\mu}=0$ and conservation law $k^{\prime}=-\left(k+k_{0}\right)$. The latter can be used to construct the reducible part of the amplitude with the following recipe. Let us start from the one-photon two-scalar amplitude

$$
\mathcal{D}^{(1)}\left(p, p^{\prime} ; \varepsilon^{\prime}, k^{\prime}\right)=e \varepsilon^{\prime} \cdot\left(p^{\prime}-p\right),
$$

which can be easily read off from (10). It yields the reducible part of the one-photon one-graviton two-scalar amplitude by simply multiplying expressions (29) and (30), and using the replacement rule 


$$
\varepsilon^{\prime \alpha} \varepsilon^{\prime \beta} \longrightarrow \frac{\delta^{\alpha \beta}}{k^{\prime 2}},
$$

which is the photon propagator in the Feynman gauge. By renaming photon polarization and momentum as $\varepsilon_{1}$ and $k_{1}$, we thus get

$$
\begin{aligned}
& \mathcal{D}_{\text {red }}^{(1,1)}\left(p, p^{\prime} ; \varepsilon_{1}, k_{1} ; \epsilon, k_{0}\right) \\
& \quad=e \kappa\left(p^{\prime}-p\right)_{\mu} \frac{\varepsilon_{1}^{\mu}\left(k_{1} \epsilon k_{1}\right)+\left(\varepsilon_{1} \epsilon\right)^{\mu} k_{1} \cdot k_{0}-k_{1}^{\mu}\left(\varepsilon_{1} \epsilon k_{1}\right)-\left(k_{1} \epsilon\right)^{\mu} \varepsilon_{1} \cdot k_{0}}{2 k_{1} \cdot k_{0}} .
\end{aligned}
$$

In other words we can obtain the latter as

$$
\mathcal{D}_{\text {red }}^{(1,1)}\left(p, p^{\prime} ; \varepsilon_{1}, k_{1} ; \epsilon, k_{0}\right)=\mathcal{D}^{(1)}\left(p, p^{\prime} ; v_{1}, k_{1}+k_{0}\right),
$$

i.e., by starting from (30) and performing the replacement

$$
\begin{aligned}
& \varepsilon_{1}^{\mu} \rightarrow v_{1}^{\mu}:=\kappa \frac{\varepsilon_{1}^{\mu}\left(k_{1} \epsilon k_{1}\right)+\left(\varepsilon_{1} \epsilon\right)^{\mu} k_{1} \cdot k_{0}-k_{1}^{\mu}\left(\varepsilon_{1} \epsilon k_{1}\right)-\left(k_{1} \epsilon\right)^{\mu} \varepsilon_{1} \cdot k_{0}}{2 k_{1} \cdot k_{0}}, \\
& k_{1}^{\mu} \rightarrow k_{1}^{\mu}+k_{0}^{\mu},
\end{aligned}
$$

note that (34) is transversal upon the replacement $\varepsilon_{1} \rightarrow k_{1}$. The rule above can be obviously extended to the $N$-photon two-scalar amplitude constructed above in (10), which thus yields the following reducible contribution

$$
\begin{aligned}
& \mathcal{D}_{\text {red }}^{(N, 1)}\left(p, p^{\prime} ; \varepsilon_{1}, k_{1}, \ldots, \varepsilon_{N}, k_{N} ; \epsilon, k_{0}\right) \\
& \quad=\sum_{i=1}^{N} \mathcal{D}^{(N)}\left(p, p^{\prime} ; \varepsilon_{1}, k_{1}, \ldots, v_{i}, k_{i}+k_{0}, \ldots \varepsilon_{N}, k_{N}\right) .
\end{aligned}
$$

Thus, the full tree-level amplitude with $N$ photons, one graviton and two scalars reads

$$
\begin{aligned}
\mathcal{D}^{(N, 1)}\left(p, p^{\prime} ; \varepsilon_{1}, k_{1}, \ldots, \varepsilon_{N}, k_{N} ; \epsilon, k_{0}\right) \\
=\mathcal{D}_{\text {irred }}^{(N, 1)}\left(p, p^{\prime} ; \varepsilon_{1}, k_{1}, \ldots, \varepsilon_{N}, k_{N} ; \epsilon, k_{0}\right) \\
\quad+\sum_{l=1}^{N} \mathcal{D}^{(N)}\left(p, p^{\prime} ; \varepsilon_{1}, k_{1}, \ldots, v_{l}, k_{l}+k_{0}, \ldots \varepsilon_{N}, k_{N}\right),
\end{aligned}
$$

where $\mathcal{D}_{\text {irred }}^{(N, 1)}$ is given by eq. (20) 'truncated' on the external scalar lines. For completeness, let us give the explicit expression for the reducible part of the amplitude with two photons. Let us start from the scalar Compton scattering amplitude, which can be easily obtained from (10) and reads

$$
\begin{aligned}
\mathcal{D}^{(2)}\left(p, p^{\prime} ; \varepsilon_{1}, k_{1}, \varepsilon_{2}, k_{2}\right) & =(-i e)^{2}\left\{2 \varepsilon_{1} \cdot \varepsilon_{2}-\frac{\varepsilon_{1} \cdot\left(p^{\prime}-p-k_{2}\right) \varepsilon_{2} \cdot\left(p^{\prime}-p+k_{1}\right)}{\left(p^{\prime}+k_{1}\right)^{2}+m^{2}}\right. \\
& \left.-\frac{\varepsilon_{1} \cdot\left(p^{\prime}-p+k_{2}\right) \varepsilon_{2} \cdot\left(p^{\prime}-p-k_{1}\right)}{\left(p^{\prime}+k_{2}\right)^{2}+m^{2}}\right\} .
\end{aligned}
$$

By applying the replacement rule given above we get 


$$
\begin{aligned}
& \mathcal{D}_{r e d}^{(2,1)}\left(p, p^{\prime} ; \varepsilon_{1}, k_{1}, \varepsilon_{2}, k_{2} ; \epsilon, k_{0}\right) \\
&= \mathcal{D}^{(2)}\left(p, p^{\prime} ; v_{1}, k_{1}+k_{0}, \varepsilon_{2}, k_{2}\right)+\mathcal{D}^{(2)}\left(p, p^{\prime} ; \varepsilon_{1}, k_{1}, v_{2}, k_{2}+k_{0}\right) \\
&= \kappa(-i e)^{2}\left\{\frac{2}{\left(k_{1}+k_{0}\right)^{2}}\left(\varepsilon_{1}^{\mu}\left(k_{1} \epsilon k_{1}\right)+\left(\varepsilon_{1} \epsilon\right)^{\mu} k_{1} \cdot k_{0}-k_{1}^{\mu}\left(\varepsilon_{1} \epsilon k_{1}\right)-\left(k_{1} \epsilon\right)^{\mu} \varepsilon_{1} \cdot k_{0}\right) \varepsilon_{2 \mu}\right. \\
&-\frac{\varepsilon_{2} \cdot\left(p^{\prime}-p+k_{1}+k_{0}\right)}{\left(p^{\prime}+k_{1}+k_{0}\right)^{2}+m^{2}} \frac{\varepsilon_{1}^{\mu}\left(k_{1} \epsilon k_{1}\right)+\left(\varepsilon_{1} \epsilon\right)^{\mu} k_{1} \cdot k_{0}-k_{1}^{\mu}\left(\varepsilon_{1} \epsilon k_{1}\right)-\left(k_{1} \epsilon\right)^{\mu} \varepsilon_{1} \cdot k_{0}}{\left(k_{1}+k_{0}\right)^{2}} \\
& \times\left(p^{\prime}-p-k_{2}\right)_{\mu} \\
&-\frac{\varepsilon_{2} \cdot\left(p^{\prime}-p-k_{1}-k_{0}\right)}{\left(p^{\prime}+k_{2}\right)^{2}+m^{2}} \frac{\varepsilon_{1}^{\mu}\left(k_{1} \epsilon k_{1}\right)+\left(\varepsilon_{1} \epsilon\right)^{\mu} k_{1} \cdot k_{0}-k_{1}^{\mu}\left(\varepsilon_{1} \epsilon k_{1}\right)-\left(k_{1} \epsilon\right)^{\mu} \varepsilon_{1} \cdot k_{0}}{\left(k_{1}+k_{0}\right)^{2}} \\
&\left.\times\left(p^{\prime}-p+k_{2}\right)_{\mu}+(1 \leftrightarrow 2)\right\} .
\end{aligned}
$$

Below, in Section 4, we test the master formula (37) by checking the on-shell transversality conditions in the photon lines and graviton line. However, to conclude the present section, let us briefly review a factorization property that links graviton-photon amplitudes to photon amplitudes.

\subsection{On-shell factorization property for the graviton photoproduction amplitude}

For a mixed scattering with one graviton and one photon, i.e. for the graviton photoproduction process, the full amplitude involving both the irreducible contributions (26) and the reducible contribution (32), on-shell factorizes in terms of the corresponding QED Compton amplitude. It can be easily seen by adopting the decomposition

$$
\epsilon^{\mu \nu} \rightarrow \epsilon^{\mu} \epsilon^{v}
$$

which yields,

$$
\begin{aligned}
& \mathcal{M}^{(1,1)}\left(p, p^{\prime} ; \varepsilon_{1}, k_{1} ; \epsilon, k_{0}\right) \\
& \quad=\frac{\kappa e}{k_{0} \cdot k_{1}}\left[\epsilon \cdot p^{\prime} k_{0} \cdot p-\epsilon \cdot p k_{0} \cdot p^{\prime}\right]\left[\frac{\varepsilon_{1} \cdot p^{\prime} \epsilon \cdot p}{p^{\prime} \cdot k_{1}}+\frac{\varepsilon_{1} \cdot p \epsilon \cdot p^{\prime}}{p^{\prime} \cdot k_{0}}+\epsilon \cdot \varepsilon_{1}\right] \\
& =H \mathcal{M}^{(2)}\left(p, p^{\prime} ; \epsilon, k_{0}, \varepsilon_{1}, k_{1}\right),
\end{aligned}
$$

where

$$
H=-\frac{\kappa}{2 e} \frac{\epsilon \cdot p^{\prime} k_{0} \cdot p-\epsilon \cdot p k_{0} \cdot p^{\prime}}{k_{0} \cdot k_{1}},
$$

and $\mathcal{M}^{(1,1)}\left(p, p^{\prime} ; \varepsilon_{1}, k_{1} ; \epsilon, k_{0}\right)$ and $\mathcal{M}^{(2)}\left(p, p^{\prime} ; \epsilon, k_{0}, \varepsilon_{1}, k_{1}\right)$ are respectively the on-shell versions of the graviton photoproduction amplitude and of the scalar QED Compton scattering given in Eq. (38). This factorization property was already studied in [36-38,40,41], and seems to be universal for four-body amplitudes with massless gauge bosons. However, beyond the four-particle level, such factorization property is not expected to hold due to the lack of enough conservation laws [36]. 


\section{Ward identities and on-shell transversality}

The dressed propagator described above in (11) is covariant upon $U(1)$ gauge transformations and invariant under diffeomorphisms. The former is described by

$$
\left\langle\phi\left(x^{\prime}\right) \bar{\phi}(x)\right\rangle_{A, g} \rightarrow\left\langle\tilde{\phi}\left(x^{\prime}\right) \tilde{\bar{\phi}}(x)\right\rangle_{\tilde{A}, \tilde{g}}=e^{i e\left(\alpha(x)-\alpha\left(x^{\prime}\right)\right)}\left\langle\phi\left(x^{\prime}\right) \bar{\phi}(x)\right\rangle_{A, g} .
$$

Using that $\delta A_{\mu}=\partial_{\mu} \alpha$, the infinitesimal part of (43) becomes the electromagnetic Ward identity generator

$$
\left[\partial_{\mu}^{y} \frac{\delta}{\delta A_{\mu}(y)}+i e\left(\delta(y-x)-\delta\left(y-x^{\prime}\right)\right)\right]\left\langle\phi\left(x^{\prime}\right) \bar{\phi}(x)\right\rangle_{A, g}=0,
$$

which holds off-shell. In momentum space, it yields an infinite set of Ward identities

$$
\begin{aligned}
\tilde{D}^{(N, 1)}\left(p, p^{\prime} ;-i k, k, \varepsilon_{1}, k_{1}, \ldots ; \epsilon, k_{0}\right) & =-i e\left[\tilde{D}^{(N-1,1)}\left(p+k, p^{\prime} ; \varepsilon_{1}, k_{1}, \ldots ; \epsilon, k_{0}\right)\right. \\
& \left.-\tilde{D}^{(N-1,1)}\left(p, p^{\prime}+k ; \varepsilon_{1}, k_{1}, \ldots ; \epsilon, k_{0}\right)\right],
\end{aligned}
$$

which can be easily tested with the special cases singled out in the section 3.1. On the other hand, on the scalar mass-shell the contact terms present in (44) do not have the correct pole structure and drop out upon truncation, whereas the first term leads to the on-shell transversality condition

$$
\mathcal{M}_{\text {irred }}^{(N, 1)}\left(p, p^{\prime} ; \varepsilon_{1}, k_{1}, \ldots,-i k_{l}, k_{l}, \ldots ; \epsilon, k_{0}\right)=0,
$$

which holds for any photon line. As before $\mathcal{M}$ is the on-shell limit of $\mathcal{D}$. Moreover, the gauge invariance of scalar QED (in curved space) ensures that the full amplitude is transversal, i.e. the reducible part of the amplitude must result separately transversal. Indeed, given that (34) vanishes upon the replacement $\varepsilon_{1} \rightarrow k_{1}$, this is enough to prove the transversality of the reducible part of the amplitude (3.2), as can easily be checked for the expression (39).

Under infinitesimal diffeomorphisms, $x^{\mu} \rightarrow x^{\mu}-\xi^{\mu}(x)$, the dressed propagator transforms as

$$
\begin{aligned}
\left\langle\tilde{\phi}\left(x^{\prime}\right) \tilde{\bar{\phi}}(x)\right\rangle_{\tilde{A}, \tilde{g}}= & \left\langle\phi\left(x^{\prime}\right) \bar{\phi}(x)\right\rangle_{A, g} \\
& +\int d^{4} y \xi^{\mu}(y)\left(\delta^{(4)}(y-x) \partial_{\mu}+\delta^{(4)}\left(y-x^{\prime}\right) \partial_{\mu}^{\prime}\right)\left\langle\phi\left(x^{\prime}\right) \bar{\phi}(x)\right\rangle_{A, g} .
\end{aligned}
$$

However, using the worldline representation (11), one can as well get

$$
\begin{aligned}
& \left\langle\tilde{\phi}\left(x^{\prime}\right) \tilde{\bar{\phi}}(x)\right\rangle_{\tilde{A}, \tilde{g}}=\left\langle\phi\left(x^{\prime}\right) \bar{\phi}(x)\right\rangle_{A, g} \\
& +\int d^{4} y\left[2 \nabla_{\mu} \xi_{\nu}(y) \frac{\delta}{\delta g_{\mu \nu}(y)}+\left(\xi^{\alpha} \partial_{\alpha} A_{\mu}(y)+\partial_{\mu} \xi^{\alpha} A_{\alpha}(y)\right) \frac{\delta}{\delta A_{\mu}(y)}\right]\left\langle\phi\left(x^{\prime}\right) \bar{\phi}(x)\right\rangle_{A, g},
\end{aligned}
$$

which, after some straightforward algebra and using expression (44), can be reduced to

$$
\begin{aligned}
& {\left[-\nabla_{\mu}^{y} \frac{2 g_{\nu \alpha}}{\sqrt{g}} \frac{\delta}{\delta g_{\mu \nu}(y)}\right.} \\
& \left.+\frac{1}{\sqrt{g}}\left(F_{\alpha \mu} \frac{\delta}{\delta A_{\mu}(y)}-\delta^{(4)}(y-x) \bar{D}_{\alpha}-\delta^{(4)}\left(y-x^{\prime}\right) D_{\alpha}^{\prime}\right)\right]\left\langle\phi\left(x^{\prime}\right) \bar{\phi}(x)\right\rangle_{A, g}=0,
\end{aligned}
$$


which is the diffeomorphism Ward identity generator. Once again there are contact terms which drop out on the scalar particle mass-shell. The two left-over terms both contribute on-shell and thus the irreducible part of the $N$-photon one-graviton amplitude is not, by itself, transversal on the graviton line; rather it fulfills, even on-shell, an inhomogeneous Ward identity. Introducing the field strength tensor $f_{i}^{\mu \nu}:=k_{i}^{\mu} \varepsilon_{i}^{\nu}-\varepsilon_{i}^{\mu} k_{i}^{\nu}$ for each photon leg, and an "effective" photon polarization vector

$$
\tilde{\varepsilon}_{i}:=\kappa f_{i} \cdot \xi,
$$

this identity can be written concisely as follows (the same identity holds for the closed-loop case [39])

$$
\tilde{D}^{(N, 1)}\left(p, p^{\prime} ; \varepsilon_{1}, k_{1}, \ldots ; k_{0} \xi, k_{0}\right)=\sum_{i=1}^{N} \tilde{D}^{(N, 0)}\left(p, p^{\prime} ; \varepsilon_{1}, k_{1}, \ldots, \tilde{\varepsilon}_{i}, k_{i}+k_{0}, \ldots, \varepsilon_{N}, k_{N}\right) .
$$

Here we have written the transformation of the (transverse traceless) polarization tensor as

$$
\epsilon_{\mu \nu} \rightarrow \epsilon_{\mu \nu}+k_{0 \mu} \xi_{v}+k_{0 \nu} \xi_{\mu}, \quad k_{0} \cdot \xi=k_{0}^{2}=0
$$

and used $k_{0} \xi$ just a shortcut notation for the symmetrized product of the two vectors. However, the full amplitude is expected to be transversal on-shell, i.e.,

$$
\mathcal{M}^{(N, 1)}\left(p, p^{\prime} ; \varepsilon_{1}, k_{1}, \ldots, \varepsilon_{N}, k_{N} ; k_{0} \xi, k_{0}\right)=0 .
$$

Using the "tree replacement" rule (34), it can be seen quite easily how this comes about: applying the transformation (52) to $v_{i}^{\mu}$, the result can be written as

$$
v_{i}^{\mu} \rightarrow-\tilde{\varepsilon}_{i}^{\mu}+\kappa \frac{k_{0} \cdot f_{i} \cdot \xi}{2 k_{i} \cdot k_{0}}\left(k_{0}+k_{i}\right)^{\mu}
$$

The second term in brackets will drop out when inserted into the photon amplitude because of the transversality in the photon polarizations. The first one will cancel the contribution of the $i$ th term on the right-hand side of (36) to the Ward identity. In the Appendix B we single out a few detailed examples.

\section{Conclusions and outlook}

We described a novel worldline approach to the computation of the tree level scattering amplitudes associated to the scalar line coupled to electromagnetism and gravity with all external legs off-shell. In particular, we provided a convenient parametrization for the graviton polarization and a replacement rule, which allowed us to easily compute amplitudes with an arbitrary number of photons and one graviton. The on-shell transversality of the amplitudes was explicitly checked.

A priori, our technique can be as well implemented to compute amplitudes with an arbitrary number of gravitons. However, in that case more care is needed in the treatment of chains of contractions between the Lee-Yang ghost fields that represent the non trivial measure [46,47].

On the other hand amplitudes with gravitons have always been the subject of extensive studies. In particular, theorems which involve gravitons with low momentum have long been analyzed [48] and, in the recent past, various soft-graviton theorems—see e.g. Ref. [49]-have been studied, due to their connections to the infrared structure of gauge theory and gravity [50]. 


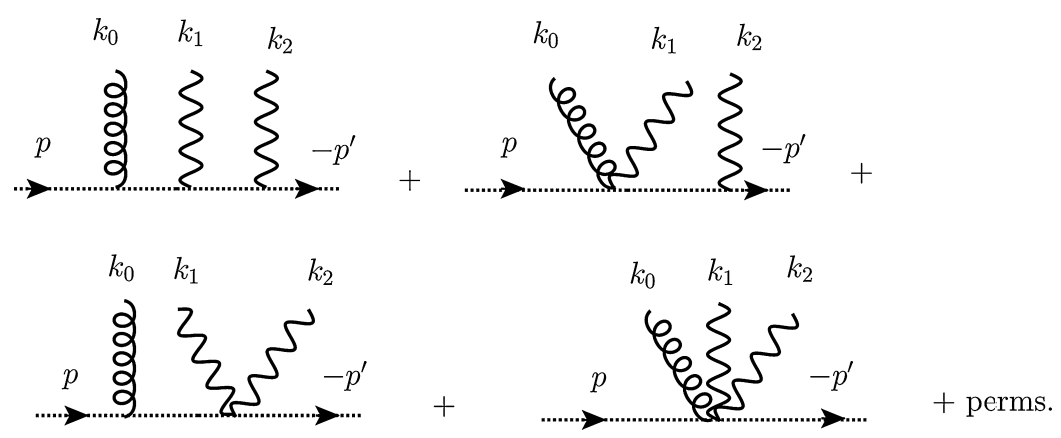

Fig. 3. Irreducible contributions to the amplitude with two-photon one-graviton, which are shown here in momentum space. 'Perms' refers to permutations between the photon lines and among the emission points. The last type of diagram, where photons and graviton are all emitted at the same point, is obviously unique.

The present manuscript wishes to provide a novel approach towards the computation of amplitudes with gravitons, which may shed new light on the structure of such quantities. In fact, our approach does not, a priori, require gravitons to have low-momentum. However, it would be helpful to reconstruct soft graviton theorems from the worldline view point, by suitably implementing from the beginning the low-momentum condition into the graviton vertex operators (13). Yet, the parametrization described in Section 3.1, which allows to simplify the computation of the worldline correlators, keeps holding for each graviton vertex operator.

\section{Declaration of competing interest}

The authors certify that they have NO affiliations with or involvement in any organization or entity with any financial interest (such as honoraria; educational grants; participation in speakers' bureaus; membership, employment, consultancies, stock ownership, or other equity interest; and expert testimony or patent-licensing arrangements), or non-financial interest (such as personal or professional relationships, affiliations, knowledge or beliefs) in the subject matter or materials discussed in this manuscript.

\section{Acknowledgements}

The Authors would like to thank Fiorenzo Bastianelli, James Edwards and Diego Trancanelli for helpful discussions.

\section{Appendix A. Two-photon one-graviton scalar propagator}

We use the master formula (20) to compute the two-photon one-graviton scalar propagator, and the related (irreducible) part of the two-photon one-graviton two-scalar amplitude, whose Feynman diagrams are depicted in Fig. 3.

It reduces to,

$$
\begin{aligned}
& \widetilde{D}^{(2,1)}\left(p, p^{\prime} ; \varepsilon_{1}, k_{1}, \varepsilon_{2}, k_{2} ; \epsilon, k_{0}\right)=(-i e)^{2}\left(-\frac{\kappa}{4}\right) \int_{0}^{\infty} d T e^{-T\left(m^{2}+p^{\prime 2}\right)} \int_{0}^{T} d \tau_{0} \int_{0}^{T} d \tau_{1} \int_{0}^{T} d \tau_{2} \\
& \times e^{\left(p^{\prime}-p\right) \cdot\left(-k_{0} \tau_{0}-k_{1} \tau_{1}-k_{2} \tau_{2}+i \varepsilon_{0}+i \varepsilon_{1}+i \varepsilon_{2}\right)} e^{k_{0} \cdot k_{1}\left|\tau_{0}-\tau_{1}\right|+k_{0} \cdot k_{2}\left|\tau_{0}-\tau_{2}\right|+k_{1} \cdot k_{2}\left|\tau_{1}-\tau_{2}\right|}
\end{aligned}
$$




$$
\begin{aligned}
& \times e^{i\left(\varepsilon_{1} \cdot k_{0}-\varepsilon_{0} \cdot k_{1}\right) \operatorname{sgn}\left(\tau_{0}-\tau_{1}\right)+i\left(\varepsilon_{2} \cdot k_{0}-\varepsilon_{0} \cdot k_{2}\right) \operatorname{sgn}\left(\tau_{0}-\tau_{2}\right)+i\left(\varepsilon_{2} \cdot k_{1}-\varepsilon_{1} \cdot k_{2}\right) \operatorname{sgn}\left(\tau_{1}-\tau_{2}\right)} \\
& \times\left. e^{2\left[\varepsilon_{0} \cdot \varepsilon_{1} \delta\left(\tau_{0}-\tau_{1}\right)+\varepsilon_{0} \cdot \varepsilon_{2} \delta\left(\tau_{0}-\tau_{2}\right)+\varepsilon_{1} \cdot \varepsilon_{2} \delta\left(\tau_{1}-\tau_{2}\right)\right]}\right|_{\text {m.l. }} .
\end{aligned}
$$

Firstly, let us consider contributions involving delta functions, which are linked to seagull diagrams. We find it convenient to 'grade' the different contributions in terms of how many delta functions occur. There is only one double-delta term (see the last diagram in Fig. 3), namely

$$
\left.e^{2} \kappa \int_{0}^{\infty} d T e^{-T\left(m^{2}+p^{\prime 2}\right)} \int_{0}^{T} d \tau_{0} e^{\tau_{0}\left(p^{\prime 2}-p^{2}\right)} \varepsilon_{0} \cdot \varepsilon_{1} \varepsilon_{0} \cdot \varepsilon_{2}\right|_{\mathrm{m} .1 .},
$$

which, using (17) and (18), reduces to

$$
\frac{1}{\left(p^{2}+m^{2}\right)\left(p^{\prime 2}+m^{2}\right)} e^{2} \kappa 2\left(\varepsilon_{1} \epsilon \varepsilon_{2}\right),
$$

whose numerator is the Feynman amplitude of the diagram where two photons and one graviton are emitted at the same point of the scalar line. Note that, also for an arbitrary number $N$ of photons - and a single graviton - this is the largest number of particles that can be emitted at the same point of the scalar line.

There are three terms with a single delta function (see the second and third diagrams and their permutations in Fig. 3), that correspond to the six Feynman diagrams where there is the emission of a pair of particles (either two photons or one photon and the graviton) from the same point of the scalar line, and the remaining particle emitted from another point on the line. Let us, for example consider the term that involves $\delta\left(\tau_{1}-\tau_{2}\right)$ (the third diagram in Fig. 3), which yield the diagrams where two photons are emitted at the same point. The integrand reads

$$
\begin{aligned}
& (-i e)^{2}\left(-\frac{\kappa}{4}\right) \varepsilon_{1} \cdot \varepsilon_{2}\left[i \varepsilon_{0} \cdot\left(p^{\prime}-p-\left(k_{1}+k_{2}\right) \operatorname{sgn}\left(\tau_{0}-\tau_{1}\right)\right)\right]^{2} \\
& \times e^{\left(p-p^{\prime}\right) \cdot\left(k_{0} \tau_{0}+\left(k_{1}+k_{2}\right) \tau_{1}\right)+k_{0} \cdot\left(k_{1}+k_{2}\right)\left|\tau_{0}-\tau_{1}\right|},
\end{aligned}
$$

which provides two diagrams, according to whether $\tau_{1}<\tau_{0}$ or $\tau_{0}<\tau_{1}$. After some straightforward algebra that corresponds to the Schwinger integral parametrization of the diagrams, we obtain

$$
\frac{1}{\left(m^{2}+p^{2}\right)\left(m^{2}+p^{\prime 2}\right)}\left(-2 e^{2} \kappa \varepsilon_{1} \cdot \varepsilon_{2}\right)\left[\frac{\left(p^{\prime} \epsilon p^{\prime}\right)}{m^{2}+\left(p^{\prime}+k_{0}\right)^{2}}+\frac{(p \epsilon p)}{m^{2}+\left(p+k_{0}\right)^{2}}\right] .
$$

Similarly, the other terms with single delta functions $\delta\left(\tau_{0}-\tau_{1}\right)$ and $\delta\left(\tau_{0}-\tau_{2}\right)$ give

$$
\begin{aligned}
\frac{1}{\left(m^{2}+p^{2}\right)\left(m^{2}+p^{\prime 2}\right)} 2 e^{2} \kappa[ & \frac{\varepsilon_{1} \cdot p\left(\varepsilon_{2} \epsilon\left(p^{\prime}-p-k_{1}\right)\right)}{m^{2}+\left(p+k_{1}\right)^{2}}+\frac{\varepsilon_{1} \cdot p^{\prime}\left(\varepsilon_{2} \epsilon\left(p-p^{\prime}-k_{1}\right)\right)}{m^{2}+\left(p^{\prime}+k_{1}\right)^{2}} \\
& \left.+\frac{\varepsilon_{2} \cdot p\left(\varepsilon_{1} \epsilon\left(p^{\prime}-p-k_{2}\right)\right)}{m^{2}+\left(p+k_{2}\right)^{2}}+\frac{\varepsilon_{2} \cdot p^{\prime}\left(\varepsilon_{1} \epsilon\left(p-p^{\prime}-k_{2}\right)\right)}{m^{2}+\left(p^{\prime}+k_{2}\right)^{2}}\right] .
\end{aligned}
$$

The term without delta functions corresponds to the leftover six Feynman diagrams where the two photons and the graviton and emitted singly by the scalar line (the first diagram in Fig. 3 and its permutations), six being the number of permutations of the three particles, which in the present worldline representation correspond to the different orderings of the three times $\tau_{i}$. The integrand in this case reads 


$$
\begin{aligned}
& (-i e)^{2}\left(-\frac{\kappa}{4}\right) \int_{0}^{\infty} d T e^{-T\left(m^{2}+p^{\prime 2}\right)} \int_{0}^{T} d \tau_{0} \int_{0}^{T} d \tau_{1} \int_{0}^{T} d \tau_{2} \\
& \times e^{\left(p^{\prime}-p\right) \cdot\left(-k_{0} \tau_{0}-k_{1} \tau_{1}-k_{2} \tau_{2}+i \varepsilon_{0}+i \varepsilon_{1}+i \varepsilon_{2}\right)} e^{k_{0} \cdot k_{1}\left|\tau_{0}-\tau_{1}\right|+k_{0} \cdot k_{2}\left|\tau_{0}-\tau_{2}\right|+k_{1} \cdot k_{2}\left|\tau_{1}-\tau_{2}\right|} \\
& \times \varepsilon_{1} \cdot\left(p^{\prime}-p+k_{0} \operatorname{sgn}\left(\tau_{0}-\tau_{1}\right)-k_{2} \operatorname{sgn}\left(\tau_{1}-\tau_{2}\right)\right) \\
& \times \varepsilon_{2} \cdot\left(p^{\prime}-p+k_{0} \operatorname{sgn}\left(\tau_{0}-\tau_{2}\right)+k_{1} \operatorname{sgn}\left(\tau_{1}-\tau_{2}\right)\right) \\
& \times \frac{1}{2}\left[\varepsilon_{0} \cdot\left(p^{\prime}-p-k_{1} \operatorname{sgn}\left(\tau_{0}-\tau_{1}\right)-k_{2} \operatorname{sgn}\left(\tau_{0}-\tau_{2}\right)\right)\right]^{2},
\end{aligned}
$$

and yields

$$
\begin{aligned}
\frac{1}{\left(m^{2}+p^{2}\right)\left(m^{2}+p^{\prime 2}\right)} 4 e^{2} \kappa & {\left[\frac{\left(p^{\prime} \epsilon p^{\prime}\right) \varepsilon_{1} \cdot\left(p+k_{2}\right) \varepsilon_{2} \cdot p}{\left(\left(p+k_{2}\right)^{2}+m^{2}\right)\left(\left(p^{\prime}+k_{0}\right)^{2}+m^{2}\right)}+(1 \leftrightarrow 2)\right.} \\
& +\frac{(p \epsilon p) \varepsilon_{1} \cdot\left(p^{\prime}+k_{2}\right) \varepsilon_{2} \cdot p^{\prime}}{\left(\left(p+k_{0}\right)^{2}+m^{2}\right)\left(\left(p^{\prime}+k_{2}\right)^{2}+m^{2}\right)}+(1 \leftrightarrow 2) \\
& \left.+\frac{\left(\left(p+k_{1}\right) \epsilon\left(p^{\prime}+k_{2}\right)\right) \varepsilon_{1} \cdot p \varepsilon_{2} \cdot p^{\prime}}{\left(\left(p+k_{1}\right)^{2}+m^{2}\right)\left(\left(p^{\prime}+k_{2}\right)^{2}+m^{2}\right)}+(1 \leftrightarrow 2)\right] .
\end{aligned}
$$

Thus,

$$
\begin{aligned}
& \mathcal{D}_{\text {irred }}^{(2,1)}\left(p, p^{\prime} ; \varepsilon_{1}, k_{1}, \varepsilon_{2}, k_{2} ; \epsilon, k_{0}\right)=\kappa e^{2}\left\{2\left(\varepsilon_{1} \epsilon \varepsilon_{2}\right)-2 \frac{\varepsilon_{1} \cdot \varepsilon_{2}\left(p^{\prime} \epsilon p^{\prime}\right)}{m^{2}+\left(p^{\prime}+k_{0}\right)^{2}}-2 \frac{\varepsilon_{1} \cdot \varepsilon_{2}(p \epsilon p)}{m^{2}+\left(p+k_{0}\right)^{2}}\right. \\
& +2 \frac{\varepsilon_{1} \cdot p\left(\varepsilon_{2} \epsilon\left(p^{\prime}-p-k_{1}\right)\right)}{m^{2}+\left(p+k_{1}\right)^{2}}+2 \frac{\varepsilon_{1} \cdot p^{\prime}\left(\varepsilon_{2} \epsilon\left(p-p^{\prime}-k_{1}\right)\right)}{m^{2}+\left(p^{\prime}+k_{1}\right)^{2}} \\
& +2 \frac{\varepsilon_{2} \cdot p\left(\varepsilon_{1} \epsilon\left(p^{\prime}-p-k_{2}\right)\right)}{m^{2}+\left(p+k_{2}\right)^{2}}+2 \frac{\varepsilon_{2} \cdot p^{\prime}\left(\varepsilon_{1} \epsilon\left(p-p^{\prime}-k_{2}\right)\right)}{m^{2}+\left(p^{\prime}+k_{2}\right)^{2}} \\
& +4 \frac{\left(p^{\prime} \epsilon p^{\prime}\right) \varepsilon_{1} \cdot\left(p+k_{2}\right) \varepsilon_{2} \cdot p}{\left(\left(p+k_{2}\right)^{2}+m^{2}\right)\left(\left(p^{\prime}+k_{0}\right)^{2}+m^{2}\right)}+4 \frac{\left(p^{\prime} \epsilon p^{\prime}\right) \varepsilon_{2} \cdot\left(p+k_{1}\right) \varepsilon_{1} \cdot p}{\left(\left(p+k_{1}\right)^{2}+m^{2}\right)\left(\left(p^{\prime}+k_{0}\right)^{2}+m^{2}\right)} \\
& +4 \frac{(p \epsilon p) \varepsilon_{1} \cdot\left(p^{\prime}+k_{2}\right) \varepsilon_{2} \cdot p^{\prime}}{\left(\left(p+k_{0}\right)^{2}+m^{2}\right)\left(\left(p^{\prime}+k_{2}\right)^{2}+m^{2}\right)}+4 \frac{(p \epsilon p) \varepsilon_{2} \cdot\left(p^{\prime}+k_{1}\right) \varepsilon_{1} \cdot p^{\prime}}{\left(\left(p+k_{0}\right)^{2}+m^{2}\right)\left(\left(p^{\prime}+k_{1}\right)^{2}+m^{2}\right)} \\
& \left.+4 \frac{\left(\left(p+k_{1}\right) \epsilon\left(p^{\prime}+k_{2}\right)\right) \varepsilon_{1} \cdot p \varepsilon_{2} \cdot p^{\prime}}{\left(\left(p+k_{1}\right)^{2}+m^{2}\right)\left(\left(p^{\prime}+k_{2}\right)^{2}+m^{2}\right)}+4 \frac{\left(\left(p+k_{2}\right) \epsilon\left(p^{\prime}+k_{1}\right)\right) \varepsilon_{2} \cdot p \varepsilon_{1} \cdot p^{\prime}}{\left(\left(p+k_{2}\right)^{2}+m^{2}\right)\left(\left(p^{\prime}+k_{1}\right)^{2}+m^{2}\right)}\right\}, \quad \text { (63) }
\end{aligned}
$$

is the irreducible part of the two-scalar two-photon one-graviton amplitude.

\section{Appendix B. Transversality of the amplitudes with one graviton and $N \leq 2$ photons}

Let us here check how the transversality of the graviton line explicitly works for $N \leq 2$. For the $N=0$ amplitude of eq. (24) we have

$$
\mathcal{M}^{(0,1)}\left(p, p^{\prime} ; k_{0} \xi, k_{0}\right)=\frac{\kappa}{2}\left(p^{\prime}-p\right) \cdot k_{0}\left(p^{\prime}-p\right) \cdot \xi,
$$

which vanishes on-sell because $k_{0}=-\left(p+p^{\prime}\right)$. For $N=1$, using on-shellness, the momentum conservation and the transversality conditions $k_{0 \mu} \epsilon^{\mu \nu}=k_{\mu} \varepsilon^{\mu}=0$, we have 


$$
\begin{aligned}
\mathcal{M}_{\text {red }}^{(1,1)}\left(p, p^{\prime} ; \varepsilon_{1}, k_{1} ; k_{0} \xi, k_{0}\right) & =-\mathcal{M}_{\text {irred }}^{(1,1)}\left(p, p^{\prime} ; \varepsilon_{1}, k_{1} ; k_{0} \xi, k_{0}\right) \\
& =e \kappa\left(p^{\prime}-p\right)_{\mu}\left(\varepsilon_{1}^{\mu} k_{1} \cdot \xi+k_{0}^{\mu} \varepsilon_{1} \cdot \xi\right),
\end{aligned}
$$

so that

$$
\mathcal{M}^{(1,1)}\left(p, p^{\prime} ; \varepsilon_{1}, k_{1} ; k_{0} \xi, k_{0}\right)=0,
$$

as expected.

The computation for the $N=2$ case is of course more complicated. However, let us sketch some details. An useful way to proceed is to identify different kind of terms in both the reducible and irreducible parts of the amplitude, that must sum up to zero separately.

Let us first consider the part of the amplitude proportional to the product $\varepsilon_{1} \cdot \varepsilon_{2}$. After performing the substitution described in Eq. (52), and denoting the corresponding reducible and irreducible contributions as $\mathcal{M}_{\text {red }}^{\varepsilon_{1} \varepsilon_{2}}$ and $\mathcal{M}_{\text {irred }}^{\varepsilon_{1} \varepsilon_{2}}$, we obtain

$$
\begin{aligned}
\mathcal{M}_{\text {irred }}^{\varepsilon_{1} \varepsilon_{2}} & =-\frac{2 \varepsilon_{1} \cdot \varepsilon_{2}}{p \cdot k_{0}}\left(p \cdot k_{0} p \cdot \xi\right)-\frac{2 \varepsilon_{1} \cdot \varepsilon_{2}}{p^{\prime} \cdot k_{0}}\left(p^{\prime} \cdot k_{0} p^{\prime} \cdot \xi\right)=-2 \varepsilon_{1} \cdot \varepsilon_{2} \xi \cdot\left(p+p^{\prime}\right), \\
\mathcal{M}_{\text {red }}^{\varepsilon_{1} \varepsilon_{2}} & =-\frac{2 \varepsilon_{1} \cdot \varepsilon_{2}}{k_{1} \cdot k_{0}}\left(k_{1} \cdot k_{0} k_{1} \cdot \xi\right)-\frac{2 \varepsilon_{1} \cdot \varepsilon_{2}}{k_{2} \cdot k_{0}}\left(k_{2} \cdot k_{0} k_{2} \cdot \xi\right)= \\
& =-2 \varepsilon_{1} \cdot \varepsilon_{2} \xi \cdot\left(k_{1}+k_{2}\right)=2 \varepsilon_{1} \cdot \varepsilon_{2} \xi \cdot\left(p+p^{\prime}\right)=-\mathcal{M}_{\text {irred }}^{\varepsilon_{1} \varepsilon_{2}},
\end{aligned}
$$

where in the last line we have used the conservation of total energy-momentum together with the transversality condition given in Eq. (52). Thus, we get

$$
\mathcal{M}_{\text {irred }}^{\varepsilon_{1} \varepsilon_{2}}+\mathcal{M}_{\text {red }}^{\varepsilon_{1} \varepsilon_{2}}=0,
$$

as expected.

Similarly we could consider the part in the total amplitude proportional to $\varepsilon_{1} \cdot \xi$, and we indicate with $\mathcal{M}_{\text {red }}^{\varepsilon_{1} \xi}$ and $\mathcal{M}_{\text {irred }}^{\varepsilon_{1} \xi}$ respectively the reducible and irreducible contributions. After some manipulations, we obtain

$$
\begin{aligned}
\mathcal{M}_{\text {irred }}^{\varepsilon_{1} \xi}= & \frac{\varepsilon_{2} \cdot p^{\prime}}{k_{2} \cdot p^{\prime}} p \cdot k_{0} \varepsilon_{1} \cdot \xi+2 \varepsilon_{1} \cdot \xi \varepsilon_{2} \cdot k_{0}+\frac{\varepsilon_{2} \cdot p^{\prime}}{k_{2} \cdot p^{\prime}}\left(p+k_{1}\right) \cdot k_{0} \varepsilon_{1} \cdot \xi \\
& +\frac{\varepsilon_{2} \cdot p}{k_{2} \cdot p} p^{\prime} \cdot k_{0} \varepsilon_{1} \cdot \xi+\frac{\varepsilon_{2} \cdot p}{k_{2} \cdot p}\left(p^{\prime}+k_{1}\right) \cdot k_{0} \varepsilon_{1} \cdot \xi \\
= & \frac{\varepsilon_{2} \cdot p^{\prime}}{k_{2} \cdot p^{\prime}} p \cdot k_{0} \varepsilon_{1} \cdot \xi+2 \varepsilon_{1} \cdot \xi \varepsilon_{2} \cdot k_{0}-\frac{\varepsilon_{2} \cdot p^{\prime}}{k_{2} \cdot p^{\prime}} p \cdot k_{1} \varepsilon_{1} \cdot \xi+\varepsilon_{1} \cdot \xi \varepsilon_{2} \cdot p^{\prime} \\
& +\frac{\varepsilon_{2} \cdot p}{k_{2} \cdot p} p^{\prime} \cdot k_{0} \varepsilon_{1} \cdot \xi-\frac{\varepsilon_{2} \cdot p}{k_{2} \cdot p} p^{\prime} \cdot k_{1} \varepsilon_{1} \cdot \xi+\varepsilon_{1} \cdot \xi \varepsilon_{2} \cdot p \\
= & \frac{\varepsilon_{2} \cdot p^{\prime}}{k_{2} \cdot p^{\prime}} \varepsilon_{1} \cdot \xi p \cdot\left(k_{0}-k_{1}\right)+\frac{\varepsilon_{2} \cdot p}{k_{2} \cdot p} \varepsilon_{1} \cdot \xi p^{\prime} \cdot\left(k_{0}-k_{1}\right)+\varepsilon_{1} \cdot \xi \varepsilon_{2} \cdot\left(k_{0}-k_{1}\right) .
\end{aligned}
$$

Notice that in the last equality we have exploited the conservation of total energy-momentum, while in the second equality we have used the relations

$$
\begin{aligned}
& k_{0} \cdot\left(p+k_{1}\right)=-p \cdot k_{1}+p^{\prime} \cdot k_{2}, \\
& k_{0} \cdot\left(p^{\prime}+k_{1}\right)=-p^{\prime} \cdot k_{1}+p \cdot k_{2} .
\end{aligned}
$$

The contribution coming from the reducible part of the amplitude is obtained as 


$$
\begin{aligned}
\mathcal{M}_{r e d}^{\varepsilon_{1} \xi}= & \frac{\varepsilon_{2} \cdot p^{\prime}}{p^{\prime} \cdot k_{2} k_{0} \cdot k_{1}} \varepsilon_{1} \cdot \xi\left(p \cdot k_{1} k_{0} \cdot k_{1}-p \cdot k_{0} k_{0} \cdot k_{1}\right)+2\left(\frac { \varepsilon _ { 1 } \cdot \xi } { 2 k _ { 0 } \cdot k _ { 1 } } \left(k_{0} \cdot k_{1} \varepsilon_{2} \cdot k_{1}\right.\right. \\
& \left.\left.-k_{0} \cdot k_{1} \varepsilon_{2} \cdot k_{0}\right)\right)+\frac{\varepsilon_{2} \cdot p}{p \cdot k_{2} k_{0} \cdot k_{1}} \varepsilon_{1} \cdot \xi\left(p^{\prime} \cdot k_{1} k_{0} \cdot k_{1}-p^{\prime} \cdot k_{0} k_{0} \cdot k_{1}\right) \\
& =-\frac{\varepsilon_{2} \cdot p^{\prime}}{k_{2} \cdot p^{\prime}} \varepsilon_{1} \cdot \xi p \cdot\left(k_{0}-k_{1}\right)-\frac{\varepsilon_{2} \cdot p}{k_{2} \cdot p} \varepsilon_{1} \cdot \xi p^{\prime} \cdot\left(k_{0}-k_{1}\right)-\varepsilon_{1} \cdot \xi \varepsilon_{2} \cdot\left(k_{0}-k_{1}\right),
\end{aligned}
$$

and the sum of the reducible and irreducible contribution vanishes, that is

$$
\mathcal{M}_{\text {irred }}^{\varepsilon_{1} \xi}+\mathcal{M}_{\text {red }}^{\varepsilon_{1} \xi}=0
$$

By Bose symmetry the contributions proportional to $\varepsilon_{2} \cdot \xi$ can be obtained from the latter with the replacements $\varepsilon_{1} \leftrightarrow \varepsilon_{2}$ and $k_{1} \leftrightarrow k_{2}$. Now we are ready to write down all the remaining terms that enter in the transversality expression for the total amplitude. We find it convenient to organize them in terms of their different denominators, which are scalar product of momenta. We thus use the notation $\mathcal{M}_{r e m}^{p k}$ to indicate those terms that have the common denominator $p \cdot k$ and similarly with others. We have,

$$
\begin{aligned}
& \mathcal{M}_{r e m}^{p^{\prime} k_{2}}=-\frac{\varepsilon_{2} \cdot p^{\prime}}{p^{\prime} \cdot k_{2}} 2 p \cdot \xi \varepsilon_{1} \cdot\left(p+k_{0}\right)+\frac{\varepsilon_{2} \cdot p^{\prime}}{p^{\prime} \cdot k_{2}} \varepsilon_{1} \cdot k_{0} p \cdot \xi+\frac{\varepsilon_{2} \cdot p^{\prime}}{p^{\prime} \cdot k_{2}} \varepsilon_{1} \cdot k_{0} \xi \cdot\left(p+k_{1}\right) \\
& +\frac{\varepsilon_{2} \cdot p^{\prime}}{p^{\prime} \cdot k_{2}} 2 \varepsilon_{1} \cdot p \xi \cdot\left(p+k_{1}\right)-\frac{\varepsilon_{2} \cdot p^{\prime}}{p^{\prime} \cdot k_{2}} 2 p \cdot \varepsilon_{1} \xi \cdot k_{1}-\frac{\varepsilon_{2} \cdot p^{\prime}}{p^{\prime} \cdot k_{2}} \varepsilon_{1} \cdot k_{0} \xi \cdot k_{1}=0, \\
& \mathcal{M}_{r e m}^{p k_{1}}=-\frac{\varepsilon_{1} \cdot p}{p \cdot k_{1}} \varepsilon_{2} \cdot k_{0} \xi \cdot\left(p+k_{1}\right)-\frac{\varepsilon_{1} \cdot p}{p \cdot k_{1}} 2 p^{\prime} \cdot \xi \varepsilon_{2} \cdot\left(p^{\prime}+k_{0}\right)+\frac{\varepsilon_{1} \cdot p}{p \cdot k_{1}} \varepsilon_{2} \cdot k_{0} \xi \cdot p^{\prime} \\
& -\frac{\varepsilon_{1} \cdot p}{p \cdot k_{1}} 2 \varepsilon_{2} \cdot p^{\prime} \xi \cdot\left(p+k_{1}\right)+\frac{\varepsilon_{1} \cdot p}{p \cdot k_{1}} 2\left(p+k_{1}\right) \cdot \varepsilon_{2} \xi \cdot k_{2}+\frac{\varepsilon_{1} \cdot p}{p \cdot k_{1}} \varepsilon_{2} \cdot k_{0} \xi \cdot k_{2} \\
& =\frac{\varepsilon_{1} \cdot p}{p \cdot k_{1}} \varepsilon_{2} \cdot k_{0} \xi \cdot\left(p^{\prime}+k_{2}\right)-\frac{\varepsilon_{1} \cdot p}{p \cdot k_{1}} 2 p^{\prime} \cdot \xi \varepsilon_{2} \cdot\left(p^{\prime}+k_{0}\right)+\frac{\varepsilon_{1} \cdot p}{p \cdot k_{1}} \varepsilon_{2} \cdot k_{0} \xi \cdot p^{\prime} \\
& +\frac{\varepsilon_{1} \cdot p}{p \cdot k_{1}} 2 \varepsilon_{2} \cdot p^{\prime} \xi \cdot\left(p^{\prime}+k_{2}\right)-\frac{\varepsilon_{1} \cdot p}{p \cdot k_{1}} 2\left(p^{\prime}+k_{0}\right) \cdot \varepsilon_{2} \xi \cdot k_{2}+\frac{\varepsilon_{1} \cdot p}{p \cdot k_{1}} \varepsilon_{2} \cdot k_{0} \xi \cdot k_{2} \\
& =0 \text {, } \\
& \mathcal{M}_{r e m}^{p^{\prime} k_{1}}=-\frac{\varepsilon_{1} \cdot p^{\prime}}{p^{\prime} \cdot k_{1}} 2 p \cdot \xi \varepsilon_{2} \cdot\left(p+k_{0}\right)+\frac{\varepsilon_{1} \cdot p^{\prime}}{p^{\prime} \cdot k_{1}} p \cdot \xi \varepsilon_{2} \cdot k_{0}-\frac{\varepsilon_{1} \cdot p^{\prime}}{p^{\prime} \cdot k_{1}} \varepsilon_{2} \cdot k_{0} \xi \cdot\left(p^{\prime}+k_{1}\right) \\
& -\frac{\varepsilon_{1} \cdot p^{\prime}}{p^{\prime} \cdot k_{1}} 2 \varepsilon_{2} \cdot p \xi \cdot\left(p^{\prime}+k_{1}\right)+\frac{\varepsilon_{1} \cdot p^{\prime}}{p^{\prime} \cdot k_{1}} 2\left(p^{\prime}+k_{1}\right) \cdot \varepsilon_{2} \xi \cdot k_{2}+\frac{\varepsilon_{1} \cdot p^{\prime}}{p^{\prime} \cdot k_{1}} \varepsilon_{2} \cdot k_{0} \xi \cdot k_{2} \\
& =-\frac{\varepsilon_{1} \cdot p^{\prime}}{p^{\prime} \cdot k_{1}} 2 p \cdot \xi \varepsilon_{2} \cdot\left(p+k_{0}\right)+\frac{\varepsilon_{1} \cdot p^{\prime}}{p^{\prime} \cdot k_{1}} p \cdot \xi \varepsilon_{2} \cdot k_{0}+\frac{\varepsilon_{1} \cdot p^{\prime}}{p^{\prime} \cdot k_{1}} \varepsilon_{2} \cdot k_{0} \xi \cdot\left(p+k_{2}\right) \\
& +\frac{\varepsilon_{1} \cdot p^{\prime}}{p^{\prime} \cdot k_{1}} 2 \varepsilon_{2} \cdot p \xi \cdot\left(p+k_{2}\right)-\frac{\varepsilon_{1} \cdot p^{\prime}}{p^{\prime} \cdot k_{1}} 2\left(p+k_{0}\right) \cdot \varepsilon_{2} \xi \cdot k_{2}+\frac{\varepsilon_{1} \cdot p^{\prime}}{p^{\prime} \cdot k_{1}} \varepsilon_{2} \cdot k_{0} \xi \cdot k_{2} \\
& =0 \text {, } \\
& \mathcal{M}_{r e m}^{p k_{2}}=-\frac{\varepsilon_{2} \cdot p}{p \cdot k_{2}} 2 p^{\prime} \cdot \xi \varepsilon_{1} \cdot\left(p^{\prime}+k_{0}\right)+\frac{\varepsilon_{2} \cdot p}{p \cdot k_{2}} \varepsilon_{1} \cdot k_{0} \xi \cdot p^{\prime}+\frac{\varepsilon_{2} \cdot p}{p \cdot k_{2}} \varepsilon_{1} \cdot k_{0} \xi \cdot\left(p^{\prime}+k_{1}\right)
\end{aligned}
$$




$$
\begin{aligned}
& +\frac{\varepsilon_{2} \cdot p}{p \cdot k_{2}} 2 \varepsilon_{1} \cdot p^{\prime} \xi \cdot\left(p^{\prime}+k_{1}\right)-\frac{\varepsilon_{2} \cdot p}{p \cdot k_{2}} 2 p^{\prime} \cdot \varepsilon_{1} \xi \cdot k_{1}-\frac{\varepsilon_{2} \cdot p}{p \cdot k_{2}} \varepsilon_{1} \cdot k_{0} \xi \cdot k_{1}=0 \\
\mathcal{M}_{r e m}^{k_{0} k_{1}}= & \frac{\varepsilon_{1} \cdot k_{0} \xi \cdot k_{1}}{k_{0} \cdot k_{1}} \varepsilon_{2} \cdot\left(k_{0}+k_{1}\right)+\frac{\varepsilon_{1} \cdot k_{0}}{k_{0} \cdot k_{1}} \varepsilon_{2} \cdot p^{\prime} \xi \cdot k_{1}+\frac{\varepsilon_{1} \cdot k_{0}}{k_{0} \cdot k_{1}} \varepsilon_{2} \cdot p \xi \cdot k_{1} \\
= & \frac{\varepsilon_{1} \cdot k_{0}}{k_{0} \cdot k_{1}} \xi \cdot k_{1} \varepsilon_{2} \cdot\left(p+p^{\prime}+k_{0}+k_{1}\right) \propto \varepsilon_{2} \cdot k_{2}=0 \\
\mathcal{M}_{r e m}^{k_{0} k_{2}}= & \frac{\varepsilon_{2} \cdot k_{0} \xi \cdot k_{2}}{k_{0} \cdot k_{2}} \varepsilon_{1} \cdot\left(k_{0}+k_{2}\right)+\frac{\varepsilon_{2} \cdot k_{0}}{k_{0} \cdot k_{2}} \varepsilon_{1} \cdot p \xi \cdot k_{2}+\frac{\varepsilon_{2} \cdot k_{0}}{k_{0} \cdot k_{2}} \varepsilon_{1} \cdot p^{\prime} \xi \cdot k_{2} \\
= & \frac{\varepsilon_{2} \cdot k_{0}}{k_{0} \cdot k_{2}} \xi \cdot k_{2} \varepsilon_{1} \cdot\left(p+p^{\prime}+k_{0}+k_{2}\right) \propto \varepsilon_{1} \cdot k_{1}=0 .
\end{aligned}
$$

Thus, all the different contributions sum up to zero and the transversality of the total amplitude is proven, i.e.,

$$
\mathcal{M}^{(2,1)}\left(p, p^{\prime} ; \varepsilon_{1}, k_{1} ; \varepsilon_{2}, k_{2} ; k_{0} \xi, k_{0}\right)=0 .
$$

What we described above is similar to what happens in flat space scalar QCD, for which a worldline approach to the computation of the $N$-gluon scalar propagator was studied in [29]: it yields the irreducible part of the $N$-gluon two-scalar amplitude. However, the non-Abelian nature of the theory implies that in order to compute the full amplitude-which is guaranteed to be transversal on the gluon lines - the latter must be completed with reducible parts [44].

\section{References}

[1] H. Elvang, Y.t. Huang, Scattering Amplitudes in Gauge Theory and Gravity, Cambridge University Press, 2015.

[2] R.P. Feynman, An operator calculus having applications in quantum electrodynamics, Phys. Rev. 84 (1951) 108.

[3] Z. Bern, D.A. Kosower, Efficient calculation of one loop QCD amplitudes, Phys. Rev. Lett. 66 (1991) 1669.

[4] Z. Bern, D.A. Kosower, The computation of loop amplitudes in gauge theories, Nucl. Phys. B 379 (1992) 451.

[5] Z. Bern, D.C. Dunbar, T. Shimada, String-based methods in perturbative gravity, Phys. Lett. B 312 (1993) 277 , arXiv:hep-th/9307001.

[6] M.J. Strassler, Field theory without Feynman diagrams: one loop effective actions, Nucl. Phys. B 385 (1992) 145 , arXiv:hep-ph/9205205.

[7] C. Schubert, Perturbative quantum field theory in the string inspired formalism, Phys. Rep. 355 (2001) 73, arXiv: hep-th/0101036.

[8] M.G. Schmidt, C. Schubert, Worldline Green functions for multiloop diagrams, Phys. Lett. B 331 (1994) 69, arXiv: hep-th/9403158.

[9] M.G. Schmidt, C. Schubert, Multiloop calculations in the string inspired formalism: the single spinor loop in QED, Phys. Rev. D 53 (1996) 2150, arXiv:hep-th/9410100.

[10] S.L. Adler, C. Schubert, Photon splitting in a strong magnetic field: recalculation and comparison with previous calculations, Phys. Rev. Lett. 77 (1996) 1695, arXiv:hep-th/9605035.

[11] M. Reuter, M.G. Schmidt, C. Schubert, Constant external fields in gauge theory and the spin 0, 1/2, 1 path integrals, Ann. Phys. 259 (1997) 313, arXiv:hep-th/9610191.

[12] H. Gies, J. Sanchez-Guillen, R.A. Vazquez, Quantum effective actions from nonperturbative worldline dynamics, J. High Energy Phys. 0508 (2005) 067, arXiv:hep-th/0505275.

[13] G.V. Dunne, C. Schubert, Worldline instantons and pair production in inhomogeneous fields, Phys. Rev. D 72 (2005) 105004, arXiv:hep-th/0507174.

[14] F. Bastianelli, A. Zirotti, Worldline formalism in a gravitational background, Nucl. Phys. B 642 (2002) 372, arXiv: hep-th/0205182.

[15] F. Bastianelli, R. Bonezzi, One-loop quantum gravity from a worldline viewpoint, J. High Energy Phys. 1307 (2013) 016, arXiv:1304.7135 [hep-th].

[16] F. Bastianelli, R. Bonezzi, O. Corradini, E. Latini, One-loop quantum gravity from the $\mathcal{N}=4$ spinning particle, J. High Energy Phys. 1911 (2019) 124, arXiv:1909.05750 [hep-th]. 
[17] F. Bastianelli, C. Schubert, One loop photon-graviton mixing in an electromagnetic field: part 1, J. High Energy Phys. 0502 (2005) 069, arXiv:gr-qc/0412095.

[18] H. Gies, K. Langfeld, L. Moyaerts, Casimir effect on the worldline, J. High Energy Phys. 0306 (2003) 018, arXiv: hep-th/0303264.

[19] F. Bastianelli, O. Corradini, E. Latini, Higher spin fields from a worldline perspective, J. High Energy Phys. 0702 (2007) 072, arXiv:hep-th/0701055.

[20] F. Bastianelli, R. Bonezzi, O. Corradini, E. Latini, Effective action for higher spin fields on (A)dS backgrounds, J. High Energy Phys. 1212 (2012) 113, arXiv:1210.4649 [hep-th].

[21] F. Bastianelli, O. Corradini, P.A.G. Pisani, Worldline approach to quantum field theories on flat manifolds with boundaries, J. High Energy Phys. 0702 (2007) 059, arXiv:hep-th/0612236.

[22] O. Corradini, J.P. Edwards, I. Huet, L. Manzo, P. Pisani, Worldline formalism for a confined scalar field, J. High Energy Phys. 1908 (2019) 037, arXiv:1905.00945 [hep-th].

[23] R. Bonezzi, O. Corradini, S.A. Franchino Vinas, P.A.G. Pisani, Worldline approach to noncommutative field theory, J. Phys. A 45 (2012) 405401, arXiv:1204.1013 [hep-th].

[24] N. Ahmadiniaz, C. Schubert, A covariant representation of the Ball-Chiu vertex, Nucl. Phys. B 869 (2013) 417, arXiv:1210.2331 [hep-ph].

[25] N. Ahmadiniaz, C. Schubert, QCD gluon vertices from the string-inspired formalism, Int. J. Mod. Phys. E 25 (2016) 1642004, arXiv:1811.10780 [hep-th].

[26] K. Daikouji, M. Shino, Y. Sumino, Bern-Kosower rule for scalar QED, Phys. Rev. D 53 (1996) 4598, arXiv:hep-ph/ 9508377.

[27] N. Ahmadiniaz, A. Bashir, C. Schubert, Multiphoton amplitudes and generalized Landau-Khalatnikov-Fradkin transformation in scalar QED, Phys. Rev. D 93 (2016) 045023, arXiv:1511.05087 [hep-ph].

[28] A. Ahmad, N. Ahmadiniaz, O. Corradini, S.P. Kim, C. Schubert, Master formulas for the dressed scalar propagator in a constant field, Nucl. Phys. B 919 (2017) 9, arXiv:1612.02944 [hep-ph].

[29] N. Ahmadiniaz, F. Bastianelli, O. Corradini, Dressed scalar propagator in a non-Abelian background from the worldline formalism, Phys. Rev. D 93 (2) (2016) 025035, Addendum: Phys. Rev. D 93 (4) (2016) 049904, arXiv: 1508.05144 [hep-th].

[30] F.A. Berezin, M.S. Marinov, Particle spin dynamics as the Grassmann variant of classical mechanics, Ann. Phys. (N. Y.) 104 (1977) 336.

[31] V.D. Gershun, V.I. Tkach, Classical and quantum dynamics of particles with arbitrary spin, JETP Lett. 29 (1979) 288.

[32] R. Bonezzi, A. Meyer, I. Sachs, Einstein gravity from the $\mathcal{N}=4$ spinning particle, J. High Energy Phys. 1810 (2018) 025, arXiv:1807.07989 [hep-th].

[33] E.S. Fradkin, D.M. Gitman, Path integral representation for the relativistic particle propagators and BFV quantization, Phys. Rev. D 44 (1991) 3230.

[34] N. Ahmadiniaz, F. Bastianelli, O. Corradini, J.P. Edwards, C. Schubert, One-particle reducible contribution to the one-loop spinor propagator in a constant field, Nucl. Phys. B 924 (2017) 377, arXiv:1704.05040 [hep-th].

[35] N. Ahmadiniaz, V.M. Banda, F. Bastianelli, O. Corradini, J.P. Edwards, C. Schubert, in preparation.

[36] C.J. Goebel, F. Halzen, J.P. Leveille, Angular zeros of Brown, Mikaelian, Sahdev, and Samuel and the factorization of tree amplitudes in gauge theories, Phys. Rev. D 23 (11) (1981) 2682.

[37] S.Y. Choi, J.S. Shim, H.S. Song, Factorization and polarization in linearized gravity, Phys. Rev. D 51 (1995) 2751 , arXiv:hep-th/9411092.

[38] B.R. Holstein, Factorization in graviton scattering and the 'natural' value of the g factor, Phys. Rev. D 74 (2006) 085002 .

[39] F. Bastianelli, O. Corradini, J.M. Dávila, C. Schubert, On the low-energy limit of one-loop photon-graviton amplitudes, Phys. Lett. B 716 (2012) 345, arXiv:1202.4502 [hep-th].

[40] N.E.J. Bjerrum-Bohr, B.R. Holstein, L. Planté, P. Vanhove, Graviton-photon scattering, Phys. Rev. D 91 (6) (2015) 064008, arXiv:1410.4148 [gr-qc].

[41] N. Ahmadiniaz, O. Corradini, J.M. Dávila, C. Schubert, Gravitational Compton scattering from the worldline formalism, Int. J. Mod. Phys. Conf. Ser. 43 (2016) 1660201.

[42] F. Bastianelli, P. van Nieuwenhuizen, Path Integrals and Anomalies in Curved Space, Cambridge University Press, Cambridge, U.K., 2006.

[43] P. Dai, Y.t. Huang, W. Siegel, Worldgraph approach to Yang-Mills amplitudes from N=2 spinning particle, J. High Energy Phys. 0810 (2008) 027, arXiv:0807.0391 [hep-th].

[44] F.M. Balli, Wordline Computation of Tree-level QED and QCD Amplitudes and Transversality, unpublished M.Sc. thesis. 
[45] F. Bastianelli, O. Corradini, P. van Nieuwenhuizen, Dimensional regularization of nonlinear sigma models on a finite time interval, Phys. Lett. B 494 (2000) 161, arXiv:hep-th/0008045.

[46] F. Bastianelli, The path integral for a particle in curved spaces and Weyl anomalies, Nucl. Phys. B 376 (1992) 113 , arXiv:hep-th/9112035.

[47] F. Bastianelli, P. van Nieuwenhuizen, Trace anomalies from quantum mechanics, Nucl. Phys. B 389 (1993) 53, arXiv:hep-th/9208059.

[48] S. Weinberg, Photons and gravitons in S-matrix theory: derivation of charge conservation and equality of gravitational and inertial mass, Phys. Rev. 135 (1964) B1049.

[49] F. Cachazo, A. Strominger, Evidence for a new soft graviton theorem, arXiv:1404.4091 [hep-th].

[50] A. Strominger, Lectures on the infrared structure of gravity and gauge theory, arXiv:1703.05448 [hep-th]. 